\title{
A finite element procedure for the analysis of thermo- mechanical solids in contact
}

\author{
Daniel Pantuso $^{\text {a }}$, Klaus-Jürgen Bathe ${ }^{\mathrm{a}, *}$, Pavel A. Bouzinov ${ }^{\mathrm{b}}$ \\ ${ }^{a}$ Department of Mechanical Engineering, Massachusetts Institute of Technology, 77 Mass Avenue, Cambridge, MA 02139, USA \\ ${ }^{\mathrm{b}}$ ADINA R \& D, Inc., Watertown, MA 02472, USA
}

Accepted 10 August 1999

\begin{abstract}
We present a finite element procedure for the analysis of fully coupled thermo-elasto-plastic response of solids including contact conditions. The continuum mechanics formulation for the solid and contact conditions is summarized and effective finite element techniques for solution are given. The constraint function method is employed to impose the contact conditions at the Gauss points of the contact surface. Other procedures widely used in finite element analysis can be considered as particular cases of the constraint function method discussed herein. (C) 2000 Elsevier Science Ltd. All rights reserved.
\end{abstract}

Keywords: Thermo-mechanical analysis; Contact; Large strains

\section{Introduction}

Inelastic analyses using finite element methods are now abundantly performed. Some basic formulations and algorithms are well-established and known to be accurate and reliable [1]. However, as successful inelastic applications have been reported, the analysis problems have become more complex. For example, today, manufacturing processes have to be analyzed in detail. Such problems involve the full coupling between the thermal, large deformation, contact, and fluid flow effects, and present a difficult but most interesting analysis challenge.

Complex inelastic analysis can only be performed effectively if formulations and solution procedures are employed that together constitute an effective analysis

\footnotetext{
* Corresponding author. Tel.: +1-617-253-6645; fax: +1617-253-2275.
}

tool. As established in earlier works, it is more effective to use a total continuum mechanics formulation instead of a Jaumann rate-type formulation. In the discretization, the finite elements used must be reliable. As clearly established, the elements must satisfy the inf-sup condition, and we largely employ the $9 / 3$ displacement/pressure based element. An efficient algorithm for elasto-plasticity must be used (we employ the effective-stress-function method) and an efficient contact solution algorithm is necessary (we employ the constraint function method). For a discussion of these items see Ref. [1].

Contact problems appear in many engineering applications, notably in metal forming operations. With the development of new algorithms and the availability of more powerful computers more sophisticated mathematical models can now be solved that allow for more insight into the physical problems.

The modeling of the contact interface requires to consider a number of very complicated processes that 
occur at the interface (a layer not thicker than 0.04 $\mathrm{mm}$ ) such as large plastic deformations. The surface roughness and the presence of impurities and other substances affect the contact behavior. Because of the physical complexity, a generally accepted contact model has not been developed as yet, but some simplified models are available.

In contact conditions, the thermal interactions between the workpiece and the tool can be very important because, for example, due to cyclic temperature changes, fatigue cracks on the surface of the tool can develop. A particularly difficult aspect of the problem is that the surfaces of the bodies entering in contact are not perfectly flat and only a small percentage of the real physical surface area is actually in contact. This effect is characterized by a heat transfer coefficient which depends, mainly, on the contact pressure and on a set of parameters that characterize the interface.

The contact physical conditions that are encountered are discussed, for example, by Rabinowicz [2], Suh [3] and Oden and Martins [4].

We present in this work the implementation of a constitutive model for thermo-mechanical contact. An earlier abridged presentation is given in Ref. [5]. The mechanical model is based on previous work, see Eterovic and Bathe [6,7] and Anand and Tong [8,9]. However, the aforementioned models do not include the thermal aspects of the problem and are therefore only applicable in isothermal processes. Our goal is to extend these models to consider situations in which the difference in temperature between the surfaces in contact, as well as the heat generated due to friction at the interface play a relevant role. Research in this area has been very active during the recent years, see for example the works of Wriggers and Miehe [10], Miles et al. [11] and Zavarise et al. [12].

The implementation of numerical procedures for contact analysis involves fundamentally two basic problems: the description of the contact interface geometry and the treatment of the constraints physically imposed in the contact problem. Also, the use of the consistent stiffness matrix, or a close approximation thereof, is important for good convergence of the Newton-Raphson algorithm.

Among the most popular procedures to numerically impose the contact conditions are the Lagrange multiplier, penalty, perturbed Lagrangian and augmented Lagrangian methods. Each of these techniques has certain attractive features, see for example Refs. [10-19].

We use in this work the constraint function method. This procedure was introduced by Eterovic and Bathe [6]. As will be shown in Section 3, some of the above

\footnotetext{
[1].
}

mentioned methods can be considered particular cases of the constraint function method presented in this work. The approach we use in the implementation of the constraint function method is that the contact variables are calculated at the Gauss points of the contactor elements.

In the next section we describe the continuum and constitutive assumptions we employ in the thermoelasto-plastic model and contact conditions. In Section 3 we then present a comparison of the approach we use to impose the contact conditions with other standard methods. In Sections 4 and 5, we focus, respectively, on our mixed finite element formulation and the numerical implementation of the contact algorithm considering the contact unknowns at the Gauss points of the contactor surface. This presentation is finally followed by the discussion of some numerical examples and the concluding remarks.

\section{Continuum model for thermo-mechanical coupling}

We present in this section the governing variational equations and the constitutive assumptions for the solids and the contact conditions ${ }^{1}$. The material model of the solids is based on previous work by Lehmann [20] and Anand [21]. The constitutive model to compute the contact heat transfer coefficient was presented by Mikic [22] and a similar law was also used in the finite element context by Wriggers and Miehe [10].

\subsection{Variational equations}

We consider a system composed of $M$ bodies $\mathscr{B}^{I}$, for $I=1, \ldots, M$. Let ${ }^{t} V^{I}$ denote the volume of body $\mathscr{B}^{I}$ at time $t$. We assume that each body $\mathscr{B}^{I}$ is an open and connected set with boundary $\partial^{t} V^{I}$ such that ${ }^{t} V^{I} \cap{ }^{t} V^{J}$ $=\varnothing$, for all $I \neq J$ and all $t \in[0, T]$. The dynamic equilibrium is enforced using the principle of virtual work,

$$
\begin{aligned}
& \sum_{I=1}^{M}\left\{\int_{{ }^{0} V^{I}} \mathbf{S} \cdot \delta \boldsymbol{\epsilon} \mathrm{d}^{0} V^{I}=\int_{{ }^{0} V^{I}} \rho\left(\mathbf{f}^{B}-\ddot{\mathbf{u}}\right) \cdot \delta \mathbf{u} \mathrm{d}^{0} V^{I}\right. \\
& \left.\quad+\int_{\partial^{0} V_{f}^{I}} \mathbf{f}^{S} \cdot \delta \mathbf{u} \mathrm{d} \partial^{0} V_{f}^{I}+\int_{\partial^{t} V_{f}^{I}} \mathbf{t}_{c} \cdot \delta \mathbf{u} \mathrm{d} \partial^{t} V_{f}^{I}\right\}
\end{aligned}
$$

referred to a previously defined reference configuration. The summation in Eq. (1) is performed over all bodies that compose the system. The tensors $\mathbf{S}$ and $\boldsymbol{\epsilon}$ denote the second Piola-Kirchhoff stress tensor and the Green-Lagrange strain tensor, respectively. The vectors $\mathbf{u}, \mathbf{f}^{B}, \mathbf{f}^{S}$ and $\mathbf{t}_{c}$ contain the displacement, body force, surface traction and contact traction components. The scalar $\rho$ represents the mass density and $\delta \mathbf{u}$ is an admissible virtual displacement field. 
In addition to being in dynamic equilibrium, the system must also be in thermodynamic equilibrium. Hence, the first law of thermodynamics or balance of energy must be satisfied. The first law can be expressed in variational form as

$$
\begin{aligned}
\sum_{I=1}^{M} & \left\{\int_{{ }^{0} V^{I}} \rho c \partial_{t} \theta \delta \theta \mathrm{d}^{0} V^{I}=\int_{{ }^{0} V^{I}} Q_{M} \delta \theta \mathrm{d}^{0} V^{I}\right. \\
& -\int_{{ }^{0} V^{I}} k \operatorname{Grad}(\theta) \cdot \operatorname{Grad}(\delta \theta) \mathrm{d}^{0} V^{I}+\int_{{ }^{0} V^{I}} \rho q^{B} \delta \theta \mathrm{d}^{0} V^{I} \\
& \left.+\int_{\partial^{0} V_{q}^{I}} q_{e}^{S} \delta \theta \mathrm{d} \partial^{0} V_{q}^{I}+\int_{\partial^{t} V_{q}^{I}} q_{c} \delta \theta \mathrm{d} \partial^{t} V_{q}^{I}\right\}
\end{aligned}
$$

where $\theta$ is the temperature field, $c$ and $k$ are the specific heat capacity and conductivity, $Q_{M}$ is the mechanical coupling term, and $q^{B}, q_{e}^{S}$ and $q_{c}$ denote, respectively, the rate of internal heat generation, the applied heat flux into the surface of the body and the heat flux due to contact interactions. Note that in Eq. (2) we use the constitutive equations to be discussed later. The second law of thermodynamics imposes restrictions on the direction of the heat transfer and has consequences on the formulation of the constitutive relations [23].

The essential boundary conditions have to be specified to complete the definition of the problem. Hence, we impose

prescribed displacements on $\partial^{0} V_{u}^{I}$

prescribed temperature on $\partial^{0} V_{\theta}^{I}$

In addition, the possibility that two or more bodies enter into contact imposes restrictions on the motion [1]. These restrictions have to be imposed onto the governing variational equations. Let $S_{c}=\partial^{t} V^{I} \cap \partial^{t} V^{J}$ be the actual surface of contact of bodies $I$ and $J$ at time $t$. We define as $S^{I J}$ that part of body $I$ capable of entering in contact with body $J$. Similarly, $S^{J I}$ is that part of body $J$ capable of entering in contact with body $I$. Notice that $S^{I J}$ and $S^{J I}$ do not need to be of equal size (see Fig. 1) in a general situation. Each pair of surfaces $S^{I J}, S^{J I}$ is called a contact pair. By convention, we call $\mathscr{B}^{I}$ the contactor body and $\mathscr{B}^{J}$ the target body. Accordingly, $S^{I J}$ is the contactor surface and $S^{J I}$ the target surface. Let us denote by $C$ the set of all
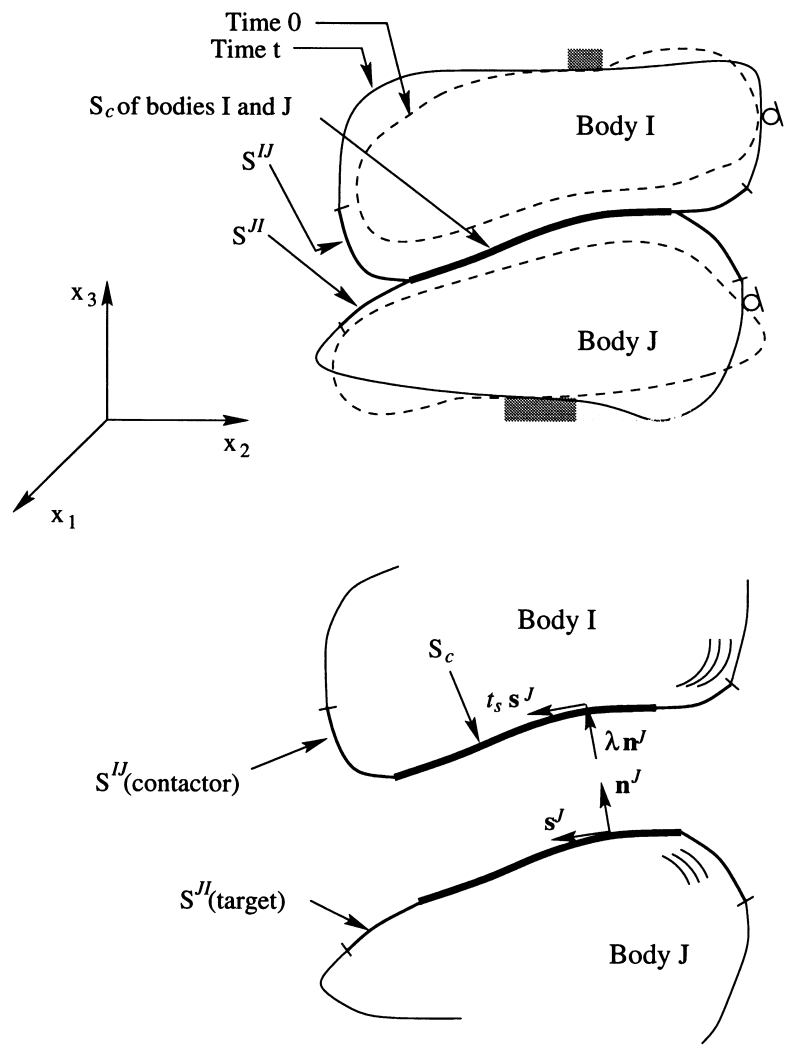

Fig. 1. Bodies in contact at time $t$. 
contact pairs,

$C=\left\{\left(S^{I J}, S^{J I}\right) ; \quad I \neq J\right\}$

Considering a contact pair $\left(S^{I J}, S^{J I}\right) \in C$, we define the scalar function $g$ on $S^{I J}$. Let $\mathbf{x}^{I} \in S^{I J}$, then

$g\left(\mathbf{x}^{I}, t\right)=\inf _{\mathbf{x}^{J} \in S^{J I}}\left\|\mathbf{x}^{I}-\mathbf{x}^{J}\right\|$

The function $g$ is called the gap function, and we have $g \geq 0$.

Assuming sufficient continuity of the contact surfaces, let $\mathbf{y}^{J} \in S^{J I}$ be the closest point to $\mathbf{x}^{I}$. Then, if $\mathbf{n}^{J}$ is the unit normal to $S^{J I}$ at $\mathbf{y}^{J}$, we have

$g\left(\mathbf{x}^{I}, t\right)=\left.\left[\mathbf{x}^{I}-\mathbf{y}^{J}\right] \cdot \mathbf{n}^{J}\right|_{\mathbf{y}^{J}}$

In expression (5), $g$ can be either positive or negative. Hence, the condition $g \geq 0$ has to be enforced. Let us denote by $\lambda$ the normal contact pressure. With the definitions given above, the conditions for the normal contact can be expressed

$g \geq 0, \lambda \geq 0, g \lambda=0$

$g q_{c}=0$

If $g>0$ there is no contact and therefore, both $\lambda$ and $q_{c}$ must vanish. On the other hand, if $g=0$ contact is present, which implies that $\lambda$ and $q_{c}$ can have a finite value different from zero. Of course, the condition $g \geq 0$ expresses that interpenetration is not allowed, and the condition $\lambda \geq 0$ enforces that no tensile contact tractions can develop. Note that heat transfer through the gap between the solids is neglected. Such heat transfer could be modeled using procedures for the analysis of coupled fluid-solid interactions [24].

Let $\mathbf{s}^{J}$ be the unit vector normal to $\mathbf{n}^{J}$ in the direction of the motion, see Fig. 1 , and $t_{s}$ be the tangential component of traction force acting onto body $I$ into the direction $\mathbf{s}^{J}$. Once contact has been established, a relative contact tangential motion is possible only if the absolute value of $t_{s}$ is sufficiently large to overcome the frictional resistance at the contact interface. This leads to the definition of the slip condition which must be satisfied pointwise on the contact surface.

Let us define the non-dimensional friction variable $\tau$ by

$\tau= \begin{cases}\frac{t_{s}}{\zeta} & \text { if } \zeta \neq 0 \\ 0 & \text { otherwise }\end{cases}$

The scalar $\zeta$ represents the slip or frictional resistance and has units of stress. For instance, when the classical Coulomb friction law is used, with $\mu$ the friction coeffi- cient,

$\zeta=\mu \lambda$

Experimental results have shown that a saturation limit exists for large values of the contact pressure. For example, in Ref. [9], Anand and Tong proposed the following equation for, $\zeta$

$\zeta=\zeta^{*} \tanh \left(\frac{\mu \lambda}{\zeta^{*}}\right)$

where $\zeta^{*}$ represents the limiting frictional resistance.

If we define the relative tangential velocity by

$v=\left(\mathbf{v}^{J}-\mathbf{v}^{I}\right) \cdot \mathbf{s}^{J}$

where $\mathbf{v}^{I}$ and $\mathbf{v}^{J}$ are the velocities of bodies $I$ and $J$ at the contact point, respectively, we have the following slip conditions,

$|\tau| \leq 1$

$|\tau|<1 \Longrightarrow v=0$

$|\tau|=1 \Longrightarrow \operatorname{sign}(v)=\operatorname{sign}(\tau)$

Eq. (12) specifies that the tangential traction cannot exceed the frictional resistance. If $\left|t_{s}\right|<\zeta$, Eq. (13) implies that the magnitude of the relative tangential velocity $v$ vanishes, while if $\left|t_{s}\right|=\zeta$ the relative tangential velocity and traction have the same sign. When Eq. (13) holds, we say that the bodies are sticking, otherwise they are slipping.

We use the constraint function method $[1,7]$ to impose the contact conditions (6) and (12)-(14). On the other hand, the contact heat transfer will be specified by means of a constitutive equation. Let $w_{n}$ be a real valued function of $g$ (the gap function) and $\lambda$ (the contact pressure) such that the solution of $w_{n}(g, \lambda)=0$ satisfies the normal contact conditions (6). Similarly, let $w_{s}$ be a real valued function of $v$ (the relative velocity) and $\tau$ (the friction variable defined by Eq. (8) such that the solution of $w_{s}(v, \tau)=0$ satisfies the slip conditions (12)-(14). Then the contact conditions are given by,

$w_{n}(g, \lambda)=0$

$w_{s}(v, \tau)=0$

Note that $w_{n}$ and $w_{s}$ are to be continuous and differentiable functions defined for all values of $g, \lambda, v$ and $\tau$.

The constraint conditions (15) and (16) can be imposed onto the governing Eq. (1) using the usual techniques. In the Lagrange multiplier method the variables $\lambda$ and $\tau$ are interpreted as Lagrange multi- 
pliers. Let $\delta \lambda$ and $\delta \tau$ be the variations of these quantities, then multiplying Eq. (15) by $\delta \lambda$ and (16) by $\delta \tau$, adding both terms and integrating over $S_{c}$, we obtain the following constraint equation

$\int_{S_{c}}\left[w_{n}(g, \lambda) \delta \lambda+w_{s}(v, \tau) \delta \tau\right] \mathrm{d} S_{c}=0$

Such equation is applicable for each contact pair $\left(S^{I J}, S^{J I}\right) \in C$.

\subsection{Constitutive model for the solid material}

We adopt the classical multiplicative decomposition of the deformation gradient $\mathbf{X}$ into the elastic part $\mathbf{X}^{e}$ and plastic part $\mathbf{X}^{p} \mathbf{X}^{p}$ to describe the kinematics at large plastic deformations $[1,25]$

$\mathbf{X}=\mathbf{X}^{e} \mathbf{X}^{p}$

We assume the existence of a Helmholtz free energy function, $\psi=\hat{\psi}\left(\mathbf{E}^{e}, \theta, s\right)$, where $\mathbf{E}^{e}=\ln \mathbf{U}^{e}, \mathbf{X}^{e}=\mathbf{R}^{e} \mathbf{U}^{e}$, and the scalar $s$, called the deformation resistance, represents an isotropic resistance to plastic flow and characterizes the inelastic response of the material. The constitutive relations for the Cauchy stress, $\tau$, and entropy, $\eta$, are then

$\overline{\boldsymbol{\tau}}=\rho \partial_{\mathbf{E}^{e}} \hat{\psi}\left(\mathbf{E}^{e}, \theta, s\right)$

$\eta=-\partial_{\theta} \hat{\psi}\left(\mathbf{E}^{e}, \theta, s\right)$

Also, the heat flow, $\mathbf{q}$, is given by

$\overline{\mathbf{q}}=\hat{\overline{\mathbf{q}}}\left(\mathbf{E}^{e}, \theta, \overline{\mathbf{g}}, s\right)$

where the vector $\mathbf{g}$ denotes the temperature gradient.

Finally, also

$\dot{s}=\hat{s}\left(\mathbf{E}^{e}, \theta, s\right)$

In the above relations, an overbar signifies that the quantity is referred to the intermediate configuration ${ }^{2}$.

As a result of the second law, we must have that

$\mathscr{D}_{\text {thermal }}=-\overline{\mathbf{q}} \cdot \overline{\mathbf{g}} \geq 0$

$\mathscr{D}_{\text {mech }}=-\rho \partial_{s} \hat{\psi} \dot{s}+\overline{\boldsymbol{\tau}} \cdot \overline{\mathbf{D}}^{p} \geq 0$

\footnotetext{
${ }^{2}$ The intermediate configuration arises as a consequence of the use of the multiplicative decomposition (Eq. (18)). See Refs. [1,25,26].

${ }^{3}$ A functional form for $\hat{\psi}^{p}$ is not required since the plastic variables are obtained by enforcing the yield condition and using the evolution Eqs. (30) and (31).
}

where $\overline{\mathbf{D}}^{p}$ is the plastic velocity strain. These relations represent thermal and mechanical conditions.

Given a yield function, $\hat{\phi}(\tau, \theta, s)$, the actual state of the body is obtained by maximizing the mechanical dissipation function subject to the constraint $\hat{\phi} \leq 0$. The first-order necessary conditions lead to the following evolution equations,

$\overline{\mathbf{D}}^{p}=\bar{\lambda} \partial_{\tau} \hat{\phi}$

$\dot{\eta}^{p}=\frac{\bar{\lambda}}{\rho} \partial_{\theta} \hat{\phi}$

where $\bar{\lambda}$ is a scalar and $\eta^{p}$ accounts for a "plastic entropy".

For metal plasticity the von Mises yield function is widely used with the yield stress $\sigma_{y}$,

$\hat{\phi}=\bar{\sigma}-\sigma_{y}$

The scalar $\bar{\sigma}$ is called the effective stress and is defined as

$\bar{\sigma}=J^{-1} \sqrt{\frac{3}{2} \overline{\boldsymbol{\tau}}^{\prime} \cdot \overline{\boldsymbol{\tau}}^{\prime}}$

where $\overline{\boldsymbol{\tau}}^{\prime}$ is the deviatoric stress and $J=\operatorname{det} \mathbf{X}$. In our studies we use the hardening law

$\sigma_{y}=\sigma_{y 0}\left\{1-w_{0}\left(\theta-\theta_{0}\right)\right\}+s$

where $\sigma_{y 0}$ is the initial yield stress when $\theta=\theta_{0}$ and $w_{0}$ is a material parameter.

The evolution equations for the plastic deformation gradient and the deformation resistance can be expressed as,

$\dot{\mathbf{X}}^{p}=\overline{\mathbf{D}}^{p} \mathbf{X}^{p}$

$\dot{s}=h\left(\theta, \dot{e}^{p}\right) \dot{e}^{p}$

The scalar $\dot{e}^{p}$ is the equivalent plastic strain,

$\dot{e}^{p}=\sqrt{\frac{2}{3} \overline{\mathbf{D}}^{p} \cdot \overline{\mathbf{D}}^{p}}$

We assume the following functional form for the Helmholtz free energy function,

$$
\begin{aligned}
\hat{\psi}\left(\mathbf{E}^{e}, \theta, s\right)= & \hat{\psi}^{\theta_{1}}(\theta)+\hat{\psi}^{\theta_{2}}\left(\theta, E_{v}^{e}\right)+\hat{\psi}^{e}\left(\mathbf{E}^{e}\right) \\
& +\hat{\psi}^{p}(\theta, s)
\end{aligned}
$$

where $^{3}$ 
$\hat{\psi}^{\theta_{1}}(\theta)=c\left\{\left(\theta-\theta_{0}\right)-\theta \log \frac{\theta}{\theta_{0}}\right\}$

$\hat{\psi}^{\theta_{2}}\left(\theta, E_{v}^{e}\right)=-3 \alpha\left(\theta-\theta_{0}\right) \partial_{E_{v}^{e}} \hat{\psi}_{V}^{e}\left(E_{v}^{e}\right)$

$\hat{\psi}_{V}^{e}\left(E_{v}^{e}\right)=\frac{\kappa}{2 \rho}\left(\operatorname{tr} \mathbf{E}^{e}\right)^{2}$

$\hat{\psi}^{e}\left(\mathbf{E}^{e}\right)=\frac{1}{\rho}\left\{G \operatorname{tr}\left(\mathbf{E}^{e 2}\right)+\frac{1}{2}\left(\kappa-\frac{2}{3} G\right)\left(\operatorname{tr} \mathbf{E}^{e}\right)^{2}\right\}$

The constants $G$ and $\kappa$ are the shear and the bulk moduli of the material. Finally, Fourier's law is used to relate the heat flux to the temperature gradient, namely,

$\overline{\mathbf{q}}=-k \overline{\mathbf{g}}$

where according to Eq. (23) we have the condition $k \geq 0$.

\subsection{Contact terms}

Now, we consider two-dimensional analysis. The extension of the mathematical model to the threedimensional case would be based on the presentation given here.

Considering the condition at time $t$, for each contact pair $\left(S^{I J}, S^{J I}\right) \in C$, the law of action and reaction implies that the contact traction acting on the target surface is equal and opposite to the contact traction acting on the contactor surface,

$\mathbf{t}_{c}^{I}+\mathbf{t}_{c}^{J}=0 \quad$ on $S_{c}$

We decompose $\mathbf{t}_{c}^{I}$ into a normal and a tangential component to the target surface according to ${ }^{4}$

$\mathbf{t}_{c}^{I}=\lambda \mathbf{n}^{J}+t_{s} \mathbf{s}^{J}$

Let $\Delta \delta \mathbf{u}^{I J}=\delta \mathbf{u}^{J}-\delta \mathbf{u}^{I}$ denote the virtual displacement of body $J$ relative to body $I$. Using Eqs. (39) and (40) the contact contribution to Eq. (1) can be written as

$$
\begin{aligned}
& \sum_{k=1}^{M} \int_{\partial^{t} V_{f}^{k}} \mathbf{t}_{c} \cdot \delta \mathbf{u} \mathrm{d} \partial^{t} V_{f}^{k} \\
& =-\sum_{\left(S^{I J}, S^{J I}\right) \in C} \int_{S_{c}}\left(\lambda \mathbf{n}^{J}+t_{S} \mathbf{s}^{J}\right) \cdot \Delta \delta \mathbf{u}^{I J} \mathrm{~d} S_{c}
\end{aligned}
$$

The first law of thermodynamics applied to a differential control volume enclosing the interface, as shown in

\footnotetext{
${ }^{4}$ We select this convention such that the normal contact traction component is always positive.
}

Fig. 2, establishes that

$q_{c}^{I}+q_{c}^{J}-q_{G}^{I J}=0 \quad$ on $S_{c}$

where $q_{G}^{I J}$ is the rate of heat per unit area generated at the interface (i.e. due to friction). The amount of heat entering body $I$ is given by,

$q_{c}^{I}=\hat{h}\left(\theta^{J}-\theta^{I}\right)+q_{G}^{I}$

where $\hat{h}$ stands for a contact heat transfer coefficient. Note that two quantities contribute to the heat transferred to body $I$. The first term on the right hand side of Eq. (43) arises because the contact surfaces are not perfectly flat, giving rise to a heat transfer resistance; in other words, the temperatures of the bodies at the interface are in general not equal. The second term represents that part of the heat generated by friction at the interface that is transferred to body $I$. The quantity $q_{G}^{I}$ can be obtained by considering the transient solution of two semi-infinite bodies abutting each other, with the quantity $q_{G}^{I J}$ accounting for the heat generated at the interface [27]. Under this assumption we have,

$q_{G}^{I}=q_{G}^{I J} \frac{k_{I}}{\xi_{I}}$

where

$\xi_{I}=\sqrt{\frac{\rho_{J} c_{J}}{\rho_{I} c_{I}}} \sqrt{k_{I} k_{J}}+k_{I}$

and $k_{K}, \rho_{K}$ and $c_{K}$ are respectively the thermal conductivity, the mass density and the specific heat capacity of body $K$, with $K=I, J$. Note that the heat flux to body $J$ is given by the same relations but interchanging the subindices $I$ and $J$.

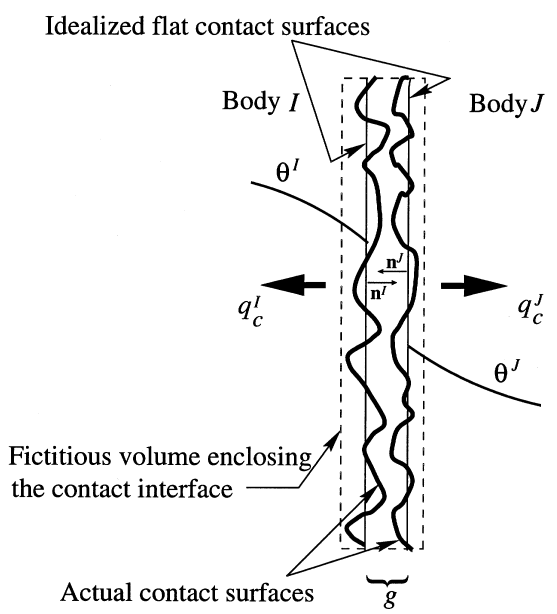

Fig. 2. Contact interface. Heat transfer analysis 
Finally, the heat generated at the interface is given

$q_{G}^{I J}=\gamma t_{s} v$

where $v$ is the relative velocity already defined in Eq. (11) and $\gamma$ is a coefficient to account for possible losses.

Now the contact heat contribution in Eq. (2) can be rewritten using Eqs. (43) and (44) in the following way,

$$
\begin{aligned}
& \sum_{I=1}^{M} \int_{\partial^{t} V_{q}^{I}} q_{c} \delta \theta \mathrm{d} \partial^{t} V_{q}^{I} \\
& =-\sum_{\left(S^{I J}, S^{J I}\right) \in C}\left\{\int_{S_{c}} \hat{h} \Delta \theta^{I J} \Delta \delta \theta^{I J} \mathrm{~d} S_{c}\right. \\
& \left.\quad-\int_{S_{c}} q_{G}^{I J} \frac{k_{I}}{\xi_{I}} \delta \theta^{I} \mathrm{~d} S_{c}-\int_{S_{c}} q_{G}^{I J} \frac{k_{J}}{\xi_{J}} \delta \theta^{J} \mathrm{~d} S_{c}\right\}
\end{aligned}
$$

where $\Delta \theta^{I J}=\theta^{J}-\theta^{I}$.

The contact zone is characterized by the contact spots through which heat transfer takes place mainly by conduction. According to Mikic [22] the heat transfer coefficient can be expressed as,

$\hat{h}=1.45 \bar{k} \frac{|\tan \delta|}{\sigma}\left\{\frac{\lambda}{H}\right\}$

where $H$ denotes the hardness of the softer material and $\lambda$ is the contact pressure. The 'mean' thermal conductivity $\bar{k}$ is given by

$\frac{1}{k}=\frac{1}{2}\left[\frac{1}{k_{I}}+\frac{1}{k_{J}}\right]$

Let $\left|\tan \delta_{I}\right|$ be the mean value (absolute value) of the slopes of the spot profile and $\sigma_{I}$ represents the standard deviation of the height of the spot profile of the interface for body $I$, then

$\tan \delta=\sqrt{\tan ^{2} \delta_{I}+\tan ^{2} \delta_{J}}$

$\sigma=\sqrt{\sigma_{I}^{2}+\sigma_{J}^{2}}$

Eq. (48) is not accurate for low contact pressures. Other models were also developed, see Ref. [28] for a summary of available models.

\section{Contact model: physical interpretation}

Our goal in this section is to give an interpretation of the contact model presented in Section 2.1. We show here that the model is physically equivalent to that presented by Anand in Ref. [8] and that, in fact, the only difference arises in the method employed to impose the constraint conditions. In addition, a comparison with other standard techniques, such as the Lagrange multiplier [14], penalty [29] and perturbed Lagrangian [17] methods, is also shortly addressed. For simplicity, we concentrate in this comparison on isothermal two-dimensional problems.

Let us first review the most relevant features of the contact model presented in Refs. [8,9]. In these papers "pseudo transient" conditions are considered, namely the rates of change of the normal and tangential contact tractions are related through constitutive relations to the relative normal and tangential velocities, $\bar{v}_{N}$ and $\bar{v}_{T}$, respectively. We have,

$\dot{\mathbf{t}}=\dot{\lambda} \mathbf{n}+\dot{t}_{s} \mathbf{s}$

$=k_{N} \overline{\mathbf{v}}_{N}+k_{T}\left(\overline{\mathbf{v}}_{T}-\overline{\mathbf{v}}_{T}^{s}\right)$

$=\left\{\mathbf{A} \overline{\mathbf{v}}-k_{T} \overline{\mathbf{v}}_{T}^{s}\right\}$

where $\overline{\mathbf{v}}_{T}^{s}$ is the slipping tangential velocity which vanishes when the process is adhering. The vector $\dot{\mathbf{t}}$ is the traction rate. The constants $k_{N}$ and $k_{T}$ can be interpreted as constitutive parameters representing a penetration modulus and a shear modulus, respectively. The second-order tensor $\mathbf{A}$ in Eq. (52) is then given by

$\mathbf{A}=k_{N} \mathbf{n n}+k_{T}(\mathbf{I}-\mathbf{n n})$

The bar over the vector $\mathbf{v}$ indicates the relative velocity between the bodies in contact. Note that the tangential component is additively decomposed into an adhering and a slipping part, $\overline{\mathbf{v}}_{T}=\overline{\mathbf{v}}_{T}^{a}+\overline{\mathbf{v}}_{T}^{s}$. The relative tangential slipping velocity is given by the slip rule,

$\overline{\mathbf{v}}_{T}^{s}=\chi\left(v-v^{a}\right) \mathbf{s}$

where $v$ was defined in Eq. (11), $v^{a}$ is the magnitude of the adhering part of the tangential velocity, and $\mathbf{s}$ gives the slip direction. The parameter $\chi$ a switching parameter indicating slipping when $\chi=1$ and adhering when $\chi=0$. The condition under which its value is 0 or 1 is given by the slip condition,

$f\left(t_{s}, \zeta\right)=t_{s}-\zeta \leq 0$

Note that the slip condition defined by Eq. (55) is equivalent to that defined by Eq. (8). The scalar $\zeta$ represents the slip resistance already defined in Eq. (8).

The time derivative of the contact traction force can be written in compact form as (see Appendix A),

$\dot{\mathbf{t}}=\mathbf{B} \overline{\mathbf{v}}$

where $\mathbf{B}$ is the non-symmetric second order tensor 
given by

$\mathbf{B}=\mathbf{A}-\frac{\chi}{k_{T}+h_{T}}\left[k_{T}^{2} \mathbf{s s}-h_{N} k_{N} k_{T} \mathbf{s n}\right]$

and

$h_{N}=\partial_{\lambda} \zeta$ and $h_{T}=\partial_{v^{s}} \zeta$

The scalar $v^{s}$ is the magnitude of the slipping part of the relative tangential velocity.

Let us suppose that the slip resistance $\zeta$ is given by Coulomb's law

$\zeta \equiv \hat{\zeta}(\lambda)=\mu \lambda$

Furthermore, we assume that the friction coefficient $\mu$ is constant. Hence, the second-order tensor $\mathbf{B}$ becomes

$\mathbf{B}^{c}=k_{N}[\mathbf{n n}+\chi \mu \mathbf{s n}]+k_{T}(1-\chi) \mathbf{s} \mathbf{s}$

and therefore we have

$\dot{\mathbf{t}}=k_{N} \overline{\mathbf{v}}_{N}+k_{T} \overline{\mathbf{v}}_{T}^{a}$

when the bodies are adhering $(\chi=0)$, and

$\dot{\mathbf{t}}=k_{N} \overline{\mathbf{v}}_{N}+\mu k_{N} \bar{v}_{N} \mathbf{S}$

when they are slipping $(\chi=1)$.

The above relations give the time rates of change of tractions as a function of velocities. In our solution algorithm we consider a particular load (or time) step. Then, if $\delta \mathbf{u}$ denotes an admissible variation of the displacement field, while in contact, the following variational equation must be satisfied for all possible $\delta \mathbf{u}$ in the displacement space $V$ considered,

$a(\mathbf{u}, \delta \mathbf{u})+\int_{S_{c}}\left(\mathbf{B}^{c} \overline{\mathbf{u}}\right) \delta \overline{\mathbf{u}} \mathrm{d} S_{c}=f(\delta \mathbf{u}) \quad \forall \delta \mathbf{u} \in V$

The bilinear form $a(\cdot, \cdot)$ and linear form $f(\cdot)$ represent the usual principle of virtual work expressions not including contact. Note that the penalty method is automatically recovered by simply interpreting the constants $k_{N}$ and $k_{T}$ as penalty parameters.

Let us next consider the contact conditions presented in Section 2.1. The governing variational equations can be written as

$$
\begin{aligned}
& a(\mathbf{u}, \delta \mathbf{u})+\int_{S_{c}}(\lambda \mathbf{n}+\tau \zeta \mathbf{s}) \cdot \Delta \delta \mathbf{u} \mathrm{d} S_{c}=f(\delta \mathbf{u}) \\
& \int_{S_{c}}\left[\delta \lambda w_{n}(\lambda, g)+\delta \tau w_{s}(\tau, v)\right] \mathrm{d} S_{c}=0
\end{aligned}
$$

Fig. 3 depicts in-plane sections of the regularized functions $w_{n_{e}}(g, \lambda)=0$ and $w_{S_{\epsilon}}(v, \tau)=0$ further discussed in Section 5.2. If the bodies are adhering, we have $|v|<$ $v^{*}$ (see Fig. 3) and we can write

$\dot{\mathbf{t}}=k_{N}^{*} \dot{g} \mathbf{n}+k_{T}^{*} v \mathbf{s}$

(where we imply the use of vectors $\mathbf{n}$ and $\mathbf{s}$ on the target body). On the other hand, if the bodies are slipping, $|\tau|=1$ and we have

$\dot{\mathbf{t}}=k_{N}^{*} \dot{g} \mathbf{n}+\mu k_{N}^{*} \dot{g} \mathbf{s}$

where the numbers $k_{N}^{*}$ and $k_{T}^{*}$ are given by

$k_{N}^{*}=-\partial_{g} \lambda$

$k_{T}^{*}=\partial_{v} \tau$

Notice that Eqs. (66) and (67) are similar to (61) and (62), respectively, and the models are physically equivalent. However, no large numbers $k_{N}^{*}$ and $k_{T}^{*}$ enter the actual implementation because we directly solve for the contact tractions, overcoming ill-conditioning problems.

The pure Lagrangian multiplier and perturbed Lagrangian methods can be obtained as particular cases of the constraint function method. Consider the frictionless situation. The Lagrange multiplier method is recovered if we use

$w_{n}(g, \lambda)=g ; \quad \lambda \geq 0$

in Eq. (15). In the same fashion, for the perturbed Lagrangian method we have to assume

$w_{n}(g, \lambda)=-\epsilon \lambda+g ; \quad \lambda \geq 0$

with $\epsilon$ a small number. The constraint function algorithm described in this paper is computationally more attractive than either the penalty method (Eq. (63)), and the pure Lagrange multiplier or perturbed Lagrangian techniques. The greater computational efficiency is due to the fact that continuous and differentiable functions $w_{n}$ and $w_{s}$ defined for any values of $g, \lambda, v$ and $\tau$ are used.

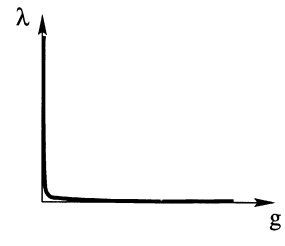

Normal contact conditions

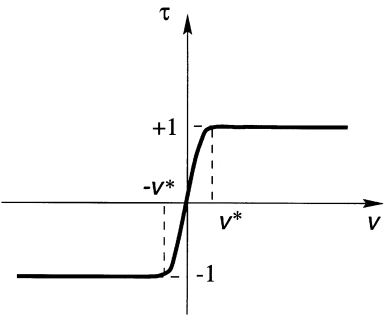

Tangential `ontact conditions
Fig. 3. Regularized functions used in the constraint fuction method. 


\section{Mixed formulation for thermo-mechanical solids}

For effective inelastic analysis it is important to use a formulation that appropriately incorporates the (almost) incompressibility constraint. An effective approach is given by the displacement/pressure (or $u / p$ ) formulation $[1,30]$.

Let $\bar{p}$ be the pressure obtained from the displacement and temperature fields,

$\bar{p}=-\frac{1}{3} \boldsymbol{\tau} \cdot \mathbf{I}$

Then the basic equations used in the $u / p$ formulation are

$$
\begin{aligned}
& \sum_{I=1}^{M} \int_{{ }^{V^{I}}}\left[\mathbf{S} \cdot \delta \boldsymbol{\epsilon}+\frac{1}{\kappa}(\bar{p}-p) \partial_{\mathbf{u}} \bar{p} \delta \mathbf{u}\right] \mathrm{d}^{0} V^{I} \\
& +\sum_{\left(S^{I J}, S^{J I}\right) \in C} \int_{S_{c}}\left(\lambda \mathbf{n}^{J}+t_{S} \mathbf{s}^{J}\right) \cdot \Delta \delta \mathbf{u}^{I J} \mathrm{~d} S_{c} \\
& =\sum_{I=1}^{M}\left\{\int_{{ }^{0} V^{I}} \rho\left(\mathbf{f}^{B}-\ddot{\mathbf{u}}\right) \cdot \delta \mathbf{u} \mathrm{d}^{0} V^{I}\right. \\
& \left.+\int_{\partial^{0} V_{f}^{I}} \mathbf{f}^{S} \cdot \delta \mathbf{u d} \partial^{0} V_{f}^{I}\right\} \\
& \begin{array}{l}
\sum_{I=1}^{M}\left\{\int_{{ }^{0} V^{I}} \rho c \partial_{t} \theta \delta \theta \mathrm{d}^{0} V^{I}-\int_{{ }^{0} V^{I}} Q_{M} \delta \theta \mathrm{d}^{0} V^{I}\right. \\
\left.\quad+\int_{{ }^{0} V^{I}} k \operatorname{Grad}(\theta) \cdot \operatorname{Grad}(\delta \theta) \mathrm{d}^{0} V^{I}\right\} \\
\quad+\sum_{\left(S^{I J}, S^{J I}\right) \in C}\left\{\int_{S_{c}} \hat{h} \Delta \theta^{I J} \Delta \delta \theta^{I J} f S_{c}\right. \\
\left.\quad-\int_{S_{c}} q_{G}^{I J} \frac{k_{I}}{\xi_{I}} \delta \theta^{I} \mathrm{~d} S_{c}-\int_{S_{c}} q_{G}^{I J} \frac{k_{J}}{\xi_{J}} \delta \theta^{J} \mathrm{~d} S_{c}\right\} \\
\quad=\sum_{I=1}^{M}\left\{\int_{{ }^{M} V^{I}} \rho q^{B} \delta \theta \mathrm{d}^{0} V^{I}+\int_{\partial^{0} V_{q}^{I}} q_{e}^{S} \delta \theta \mathrm{d} \partial^{0} V_{q}^{I}\right\}
\end{array}
\end{aligned}
$$

$\int_{{ }^{0} V^{I}} \frac{1}{\kappa}(\bar{p}-p) \delta p \mathrm{~d}^{0} V^{I}=0$

where $p$ is the separately interpolated pressure.

Using the appropriate interpolations for the displacements and the pressure, the $u / p$ formulation is optimal (with the convergence rate independent of whether a compressible or incompressible material is considered). Eqs. (17), (73)-(75) constitute a set of nonlinear equations to be solved for the displacements, pressure, temperature and the contact variables $\lambda$ and $\tau$.

\subsection{Mixed finite element interpolations}

Let $n n$ denote the number of nodes of the element. The original geometry, the total displacement and the temperature fields are defined using the standard isoparametric interpolation functions,

$\mathbf{x}=\sum_{i=1}^{n n} h_{i}(\mathbf{r}) \mathbf{x}_{i}$

$\mathbf{u}=\sum_{i=1}^{n n} h_{i}(\mathbf{r}) \mathbf{u}_{i}$

$\theta=\sum_{i=1}^{n n} h_{i}(\mathbf{r}) \theta_{i}$

In these equations, the $h_{i}$ 's are the interpolation functions, $\mathbf{r}$ indicates the isoparametric coordinates $(r, s)$, and $\mathbf{x}_{i}, \mathbf{u}_{i}$ and $\theta_{i}$ are the nodal point coordinates, displacements and temperatures, respectively.

The selection of the pressure interpolation is crucial to obtain accurate results in the solution. Let $n p$ be the number of element functions to interpolate the pressure field, then we have

$p=\sum_{i=1}^{n p} \hat{h_{i}}(\mathbf{r}) p_{i}$

where the $\hat{h}_{i}$ 's are the interpolation functions and the $p_{i}$ 's represent element pressure degrees of freedom. Once the displacement interpolations have been selected, there are different options to interpolate the pressure field. However, for an effective and optimal element behavior, the inf-sup condition [1] must be satisfied. A number of elements that satisfy the inf-sup condition are presented in Ref. [1], and a new 4-node element that satisfies the inf-sup condition was developed by Pantuso and Bathe [31]. However, this element leads to hour-glassing in the large strain range [32].

Upon discretization, the governing finite element equations are

$$
\begin{aligned}
& \mathbf{M}^{t+\Delta t} \ddot{\mathbf{u}}+{ }^{t+\Delta t} \mathbf{F}_{u}={ }^{t+\Delta t} \mathbf{R}-{ }^{t+\Delta t} \mathbf{R}_{c} \\
& \mathbf{C}^{t+\Delta t} \dot{\boldsymbol{\theta}}+{ }^{t+\Delta t} \mathbf{Q}_{\theta}={ }^{t+\Delta t} \mathbf{Q}-{ }^{t+\Delta t} \mathbf{Q}_{c} \\
& { }^{t+\Delta t} \mathbf{F}_{p}=\mathbf{0} \\
& { }^{t+\Delta t} \mathbf{F}_{c}=\mathbf{0}
\end{aligned}
$$

The resulting nonlinear system of equations is solved 
by the Newton-Raphson procedure. For impact conditions, the velocity and acceleration compatibility between the contacting bodies would be imposed as described in Ref. [33].

\subsection{Thermo-elasto-plastic material}

We use a thermo-elasto-plastic material model given in terms of the Hencky strain tensor and the corresponding work conjugate stress tensor, see Section 2. Therefore, we express our variational governing equations in terms of these quantities using,

$\int_{0^{0}} \mathbf{S} \cdot \delta \boldsymbol{\epsilon} \mathrm{d}^{0} V=\int_{{ }^{0} V} \overline{\boldsymbol{\tau}} \cdot \delta \mathbf{E} \mathrm{d}^{0} V$

Eq. (73) can now be written as

$$
\begin{aligned}
& \sum_{I=1}^{M} \int_{{ }^{0} V^{I}}\left[\overline{\boldsymbol{\tau}} \cdot \delta \mathbf{E}+\frac{1}{\kappa}(\bar{p}-p) \partial_{\mathbf{u}} \bar{p} \delta \mathbf{u}\right] \mathrm{d}^{0} V^{I} \\
& \quad+\sum_{\left(S^{I J}, S^{J I}\right) \in C} \int_{S_{c}}\left(\lambda \mathbf{n}^{J}+t_{S} \mathbf{s}^{J}\right) \cdot \Delta \delta \mathbf{u}^{I J} \mathrm{~d} S_{c} \\
& \quad=\sum_{I=1}^{M}\left\{\int_{{ }^{0} V^{I}} \rho\left(\mathbf{f}^{B}-\ddot{\mathbf{u}}\right) \cdot \delta \mathbf{u} d^{0} V^{I}\right. \\
& \left.\quad+\int_{\partial^{0} V_{f}^{I}} \mathbf{f}^{S} \cdot \delta \mathbf{u} \mathrm{d} \partial^{0} V_{f}^{I}\right\}
\end{aligned}
$$

and the mechanical coupling term, $Q_{M}$ in the energy equation is given by

$Q_{M}=\omega \overline{\boldsymbol{\tau}} \cdot \overline{\mathbf{D}}^{p}$

where $\omega$ is a parameter and we are only including the plastic work effect.

It only remains to address the integration of the evolution Eqs. (30)-(32) which define the plastic flow over the interval $[t, t+\Delta t]$. The objective is to obtain the stress tensor and the internal plastic variables that determine the final state of the material. Let us assume that we know at each Gauss point the set of variables $\left\{\mathbf{X}^{p}, \sigma_{y}\right\}_{t}$ at time $t$ and we search for $\left\{\mathbf{X}^{p}, \sigma_{y}\right\}_{t+\Delta t}$ at time $t+\Delta t$. This evaluation is both displacement and temperature driven.

\subsection{Return mapping algorithm}

Let us consider that we know the deformation gradient, $\mathbf{X}$, and the temperature, $\theta$, at time $t+\Delta t$. Then we set,

$\mathbf{X}_{*}^{e}={ }_{0}^{t+\Delta t} \mathbf{X}\left({ }_{0}^{t} \mathbf{X}^{p}\right)^{-1}$

where $\mathbf{X}_{*}^{e}$ is a trial elastic deformation gradient. Using $\mathbf{X}_{*}^{e}$ we can calculate
$\mathbf{E}_{*}^{e}=\ln \mathbf{U}_{*}^{e}$

$\mathbf{E}_{*}^{e^{\prime}}=\mathbf{E}_{*}^{e}-\operatorname{tr}\left(\mathbf{E}_{*}^{e}\right) \mathbf{I}$

$\overline{\boldsymbol{\tau}}_{*}^{\prime}=2 G \mathbf{E}_{*}^{e^{\prime}}$

$\bar{\sigma}_{*}=J^{-1} \sqrt{\frac{3}{2} \overline{\boldsymbol{\tau}}_{*}^{\prime} \cdot \overline{\boldsymbol{\tau}}_{*}^{\prime}}$

$\phi_{*}=\bar{\sigma}_{*}-\sigma_{y}$

If $\phi_{*}<0$, the incremental step is elastic and,

${ }^{t+\Delta t} \overline{\boldsymbol{\tau}}^{\prime}=\overline{\boldsymbol{\tau}}_{*}^{\prime}$

$\dot{e}^{p}=0$

${ }_{0}^{t+\Delta t} \mathbf{X}^{p}={ }_{0}^{t} \mathbf{X}^{p}$

${ }^{t+\Delta t} \sigma_{y}={ }^{t} \sigma_{y}$

On the other hand, if $\phi_{*}>0$, the trial state defined by Eqs. (87)-(92) is not admissible and a correction is required. Using the Euler backward method we have that,

$$
\begin{aligned}
{ }_{0}^{t+\Delta t} \mathbf{X}^{p} & =\exp \left(\Delta t \overline{\mathbf{D}}^{p}\right){ }^{t} \mathbf{X}^{p} \\
{ }^{t+\Delta t} \mathbf{X}^{e} & ={ }^{t+\Delta t} \mathbf{X}\left({ }_{0}^{t} \mathbf{X}^{p}\right){ }^{-1} \exp \left(-\Delta t \overline{\mathbf{D}}^{p}\right) \\
& =\mathbf{X}_{*}^{e} \exp \left(-\Delta t \overline{\mathbf{D}}^{p}\right)
\end{aligned}
$$

Note that Eq. (97) ensures that the volume is preserved during plastic flow. Following Refs. [1,34,35], the deviatoric part of the stress tensor is given by

${ }^{t+\Delta t} \overline{\boldsymbol{\tau}}^{\prime}=\overline{\boldsymbol{\tau}}_{*}^{\prime}-2 G \Delta t \overline{\mathbf{D}}^{p}$

where

$\overline{\mathbf{D}}^{p}=\sqrt{\frac{3}{2}} \dot{e}^{p t+\Delta t} \overline{\mathbf{N}}$

and ${ }^{t+\Delta t} \overline{\mathbf{N}}$ is the unit normal to the yield surface. For the particular case in which the hardening modulus $h$ is only a function of the temperature, the equivalent plastic strain is given by

${ }^{t+\Delta t} e^{p}={ }^{t} e^{p}+\frac{{ }^{t+\Delta t} J \bar{\sigma}_{*}-{ }^{t+\Delta t} J^{t+\Delta t} \sigma_{y}}{3 G+{ }^{t+\Delta t} h^{t+\Delta t} J}$

hence, 


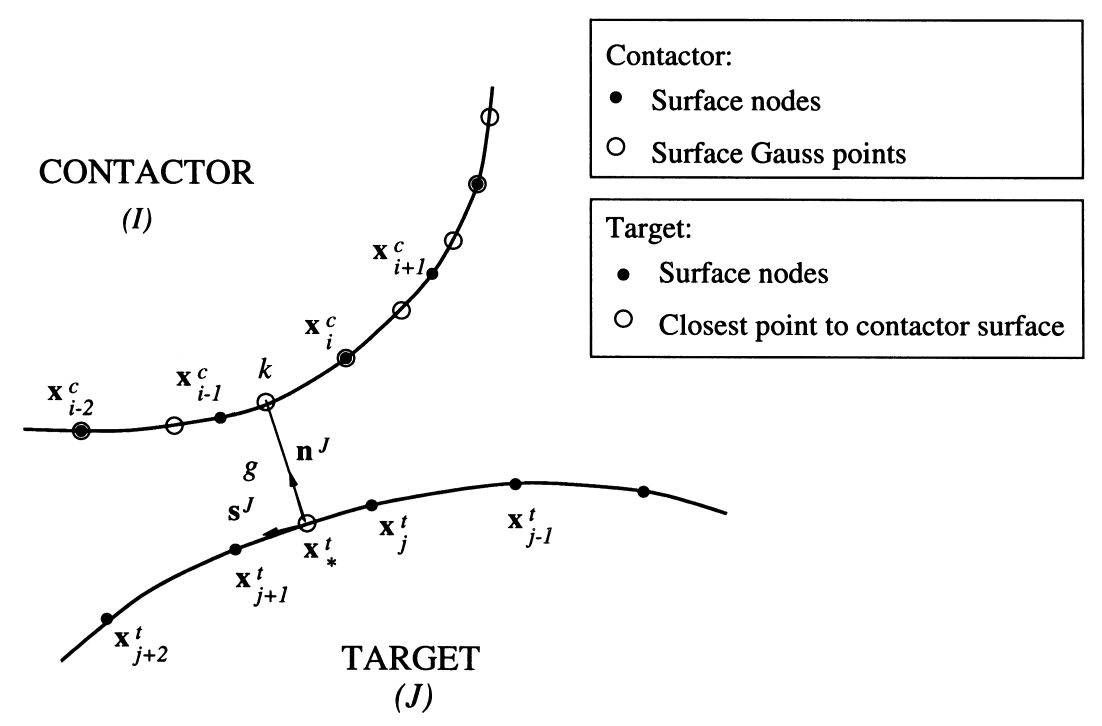

(a) Contact interface geometry

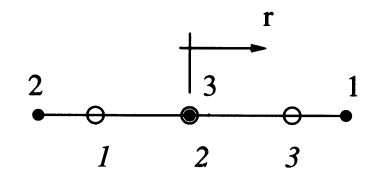

(b) Typical contactor element

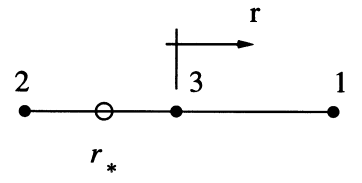

(c) Typical target element

Fig. 4. Definitions used in the discretization of the contact geometry.

${ }^{t+\Delta t} \overline{\boldsymbol{\tau}}^{\prime}=2 G\left\{1-\frac{3 G \Delta e^{p}}{{ }^{t+\Delta t} J \bar{\sigma}_{*}}\right\}{ }^{t+\Delta t} \mathbf{E}^{e^{\prime}}$

Differentiation of ${ }^{t+\Delta t} \overline{\boldsymbol{\tau}}^{\prime}$ leads to the algorithmic consistent tangent constitutive tensor, whose mechanical part is

$\mathbf{C}^{E P}=2 G\left[\gamma_{1} \mathscr{I}^{\prime}+\gamma_{2}\left(\overline{\boldsymbol{\tau}}_{*}^{\prime} \overline{\boldsymbol{\tau}}_{*}^{\prime}\right)\right]+\gamma_{1} \gamma_{3}\left(\overline{\boldsymbol{\tau}}_{*}^{\prime} \mathbf{I}\right)+\kappa(\mathbf{I I})$

and the thermal part is

$\mathbf{C}^{\theta}=\left[w_{0} \sigma_{y 0}+\Delta e^{p} \partial_{\theta} h\right] \gamma_{3} \frac{\overline{\boldsymbol{\tau}}_{*}^{\prime}}{\overline{\bar{\sigma}}_{*}}$

where the hardening as a function of $\theta$ has to be defined. The constants $\gamma_{1}$ to $\gamma_{3}$ in Eqs. (103) and (104) are given by

$\gamma_{1}=1-\frac{3 G \Delta e^{p}}{{ }^{t+\Delta t} J \bar{\sigma}_{*}}$

$$
\gamma_{2}=\frac{9 G}{2\left({ }^{t+\Delta t} J \bar{\sigma}_{*}\right)^{2}}\left\{\frac{\Delta e^{p}}{{ }^{t+\Delta t} J \bar{\sigma}_{*}}-\frac{1}{3 G+{ }^{t+\Delta t} h^{t+\Delta t} J}\right\}
$$

$$
\gamma_{3}=\frac{3 G}{3 G+{ }^{t+\Delta t} h^{t+\Delta t} J}
$$

and

$$
\mathscr{I}^{\prime}=\mathscr{I}-\frac{1}{3} \mathbf{I I}
$$

where I and $\mathscr{I}$ are the second and fourth order identity tensors, respectively.

Hence,

$\mathrm{d} \overline{\boldsymbol{\tau}}=\mathbf{C}^{E P} \mathrm{~d} \mathbf{E}^{e}+\mathbf{C}^{\theta} \mathrm{d} \theta$

The contribution to the energy equation can be evaluated using Eq. (100) to calculate $\overline{\mathbf{D}}^{p}$. The linearization required for the Newton-Raphson procedure gives, 
$\mathrm{d}\left(\overline{\boldsymbol{\tau}} \cdot \overline{\mathbf{D}}^{p}\right)=2 G \gamma_{4} \overline{\boldsymbol{\tau}}_{*} \cdot \mathrm{d} \mathbf{E}^{e^{\prime}}+\gamma_{5} \mathrm{~d} E_{v}+\gamma_{6} \mathrm{~d} \theta$

and the constants $\gamma_{4}, \gamma_{5}$ and $\gamma_{6}$ are given by,

$\gamma_{4}=\frac{3}{2}\left\{\frac{1}{3 G+{ }^{t+\Delta t} h^{t+\Delta t} J}\left[1-6 \frac{G \Delta e^{p}}{t+\Delta t} J \bar{\sigma}_{*}\right]+\frac{\Delta e^{p}}{{ }^{t+\Delta t} J \bar{\sigma}_{*}}\right\}$

$\gamma_{5}=\frac{{ }^{t+\Delta t} J \bar{\sigma}_{*}}{3 G+{ }^{t+\Delta t} h^{t+\Delta t} J}\left[9 G \Delta e^{p}-{ }^{t+\Delta t} J \bar{\sigma}_{*}-\frac{18\left(G \Delta e^{p}\right)^{2}}{{ }^{t+\Delta t} J \bar{\sigma}_{*}}\right]$

$\gamma_{6}=\frac{w_{0} \sigma_{y 0}+\Delta e^{p} \partial_{\theta} h}{3 G+{ }^{t+\Delta t} h^{t+\Delta t} J}\left[{ }^{t+\Delta t} J \bar{\sigma}_{*}-6 G \Delta e^{p}\right]^{t+\Delta t} J$

Note that for the evaluation of the consistent stiffness matrix a further transformation is required in Eq. (109) from the differential strains $\mathrm{dE}^{e}$ to the differential total displacements.

\section{Thermo-mechanical contact: numerical implementation}

Fig. 4 shows the contact surface discretization of two bodies coming into contact. Let $m^{c}$ and $m^{t}$ be the contactor and target elements to which the Gauss point $k$ and the point $\mathbf{x}_{*}^{\mathrm{t}}$ belong, respectively, and $m_{k}$ denote the pair $\left\{m^{c}, m^{t}\right\}$, which we call a contact element pair. For each contact element pair, we define

$\mathbf{U}^{m_{k}}=\left\{\mathbf{u}_{p}^{c}, \mathbf{u}_{q}^{t}\right\}$

$\boldsymbol{\Theta}^{m_{k}}=\left\{\theta_{p}^{c}, \theta_{q}^{t}\right\}$

where the indices $p$ and $q$ go from 1 to $n n c$ (number of nodes of each contactor element) and nnt (number of nodes of each target element), respectively. Hence, $\mathbf{U}^{m_{k}}$ and $\boldsymbol{\Theta}^{m_{k}}$ contain, respectively, the nodal displacements and temperatures that correspond to the contact element pair $m_{k}$. We also define for each Gauss point $k$ the vector

$\Sigma^{k}=\{\lambda, \tau\}$

which contains the unknown contact variables at the Gauss point $k$.

The contactor Gauss point ${ }^{5}$ coordinates, displacements and temperature are obtained using

\footnotetext{
${ }^{5}$ From here onwards, we refer to a contactor Gauss point simply as a contactor point.
}

$\mathbf{x}_{k}^{c}=\left.\sum_{n=1}^{n n c} h_{n}\right|_{r=r_{k}} \mathbf{x}_{n}^{c}$

$\mathbf{u}_{k}^{c}=\left.\sum_{n=1}^{n n c} h_{n}\right|_{r=r_{k}} \mathbf{u}_{n}^{c}$

$\theta_{k}^{c}=\left.\sum_{n=1}^{n n c} h_{n}\right|_{r=r_{k}} \theta_{n}^{c}$

where the $h_{n}$ 's represent the functions used for 1D isoparametric interpolations and $r_{k}$ denotes the Gauss point considered. In a similar way, once the target point $x_{*}^{t}$ in element $m^{t}$ has been determined, and hence its corresponding isoparametric coordinate $r_{*}$, we have that,

$\mathbf{u}_{*}^{t}=\left.\sum_{n=1}^{n n t} h_{n}\right|_{r=r_{*}} \mathbf{u}_{n}^{t}$

$\theta_{*}^{t}=\left.\sum_{n=1}^{n n t} h_{n}\right|_{r=r_{*}} \theta_{n}^{t}$

are the displacements and temperature evaluated at $\mathrm{x}_{*}^{t}$.

The discretized relative displacements and temperature corresponding to the contactor point $k$ are then given by

$\Delta \mathbf{u}_{k}^{I J}=\mathbf{u}_{*}^{t}-\mathbf{u}_{k}^{c}$

$\Delta \theta_{k}^{I J}=\theta_{*}^{t}-\theta_{k}^{c}$

and the corresponding virtual relative displacements and temperature are defined as

$\Delta \delta \mathbf{u}_{k}^{I J}=\delta \mathbf{u}_{*}^{t}-\delta \mathbf{u}_{k}^{c}$

$\Delta \delta \theta_{k}^{I J}=\delta \theta_{*}^{t}-\delta \theta_{k}^{c}$

The curve segment over the element $m^{t}$ is defined by

$\mathbf{Y}\left(r ; \mathbf{U}^{m_{k}}\right)=\sum_{n=1}^{n n t} h_{n}(r) \mathbf{x}_{n}^{t}$

The target point $\mathrm{x}_{*}^{t}$ is defined as the closest point to the contactor point $k$. Hence, it is obtained by solving the following problem,

$r_{*}\left(\mathbf{U}^{m_{k}}\right)=\min \left\|\mathbf{x}_{k}^{\mathrm{c}}\left(\mathbf{U}^{m_{k}}\right)-\mathbf{Y}\left(r ; \mathbf{U}^{m_{k}}\right)\right\|^{2}$

Once $r_{*}$ has been determined, the normal and tangential unit vectors at $\mathrm{x}_{*}^{t}$ can be immediately calculated using the following expressions, 
$\mathbf{s}^{J}=\frac{\left.\mathbf{Y}^{\prime}\right|_{r=r_{*}}}{\left\|\left.\mathbf{Y}^{\prime}\right|_{r=r_{*}}\right\|}$

$\mathbf{n}^{J}=\frac{\left.\mathbf{Y}^{\prime}\right|_{r=r_{*}}}{\left\|\left.\mathbf{Y}^{\prime}\right|_{r=r_{*}}\right\|} \times \mathbf{e}=\mathbf{s}^{J} \times \mathbf{e}$

where $(\cdot)^{\prime}$ denotes differentiation with respect to the isoparametric variable $r$. The unit vector $\mathbf{e}$ is normal to the $\mathbf{n}-\mathbf{s}$ plane, with $\mathbf{n}-\mathbf{s}-\mathbf{e}$ being a right-hand system.

This problem leads to the following scalar equation to be solved for $r_{*}$

$P\left(r ; \mathbf{U}^{m_{k}}\right)=\left[\mathbf{x}_{k}^{c}-\mathbf{Y}\right] \cdot \mathbf{s}^{J}=0$

where $P\left(r ; \mathbf{U}^{m_{k}}\right)$ is a polynomial of first order (for 2node target segment interpolations) or third order (for 3 -node target segment interpolations) in the variable $r$.

The gap function at $r_{*}$ is then

$g\left(r_{*} ; \mathbf{U}^{m_{k}}\right)=\left.\left[\mathbf{x}_{k}^{c}-\left.\mathbf{Y}\right|_{r=r_{*}}\right] \cdot \mathbf{n}^{J}\right|_{r=r_{*}}$

Notice the dependence of all these Eqs. (127)-(131), on the nodal displacements $\mathbf{U}^{m_{k}}$.

To obtain the consistent tangent stiffness matrix, we need

$\partial_{\mathbf{U}} \mathbf{S}^{J}=\left[\mathbf{I}-\mathbf{s}^{J} \mathbf{s}^{J}\right] \frac{\left.\partial_{\mathbf{U}} \mathbf{Y}^{\prime}\right|_{r=r_{*}}}{\left\|\left.\mathbf{Y}^{\prime}\right|_{r=r_{*}}\right\|}$

and straightforwardly,

$\partial_{\mathbf{U}} \mathbf{n}^{J}=\partial_{\mathbf{U}} \mathbf{s}^{J} \times \mathbf{e}$

The implementation of a numerical algorithm is given in Table 1 for each contactor Gauss point $k$.

\subsection{Contact finite element equations}

Fig. 5 depicts the elements involved during contact and also the corresponding local variables that have to be considered. The vectors ${ }^{t+\Delta t} \mathbf{R}_{c}$ and ${ }^{t+\Delta t} \mathbf{Q}_{c}$ are obtained by assembling for all Gauss points $k=$ $1, \ldots, K$ the local contact vectors ${ }^{t+\Delta t} \mathbf{R}_{c}^{k}$ and ${ }^{t+\Delta t} \mathbf{Q}_{c}^{k}$ given by,

${ }^{t+\Delta t} \mathbf{R}_{c}^{k}=\left[\begin{array}{l}-\left(\lambda \mathbf{n}+t_{s} \mathbf{s}\right) h_{i} \operatorname{det} w \\ \left(\lambda \mathbf{n}+t_{s} \mathbf{s}\right) h_{j}^{*} \operatorname{det} w\end{array}\right]$

${ }^{t+\Delta t} \mathbf{Q}_{c}^{k}=\left[\begin{array}{c}-\left(\hat{h} \Delta \theta^{I J}+q_{G}^{I J} \frac{k_{I}}{\xi_{I}}\right) h_{i} \operatorname{det} w \\ \left(\hat{h} \Delta \theta^{I J}-q_{G}^{I J} \frac{k_{J}}{\xi_{J}}\right) h_{j}^{*} \operatorname{det} w\end{array}\right]$

Here, $h_{i}$ and $h_{j}^{*}$ denote the interpolation functions evaluated at the Gauss point and its corresponding closest point on the target surface, $r_{*}$, and det and $w$ are the

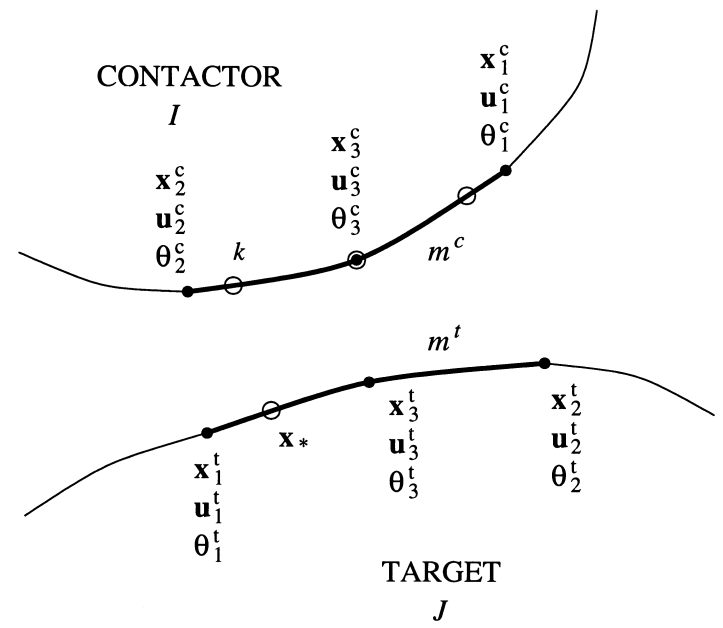

Fig. 5. Variables involved in contact analysis for each contact element pair.

Jacobian determinant and weight at point $k$, respectively.

Similarly, for each Gauss point the vector ${ }^{t+\Delta t} \mathbf{F}_{c}^{k}$ is given by

${ }^{t+\Delta t} \mathbf{F}_{c}^{k}=\left[\begin{array}{l}w_{n_{\epsilon}}(g, \lambda) \\ w_{s_{\epsilon}}(v, \tau)\end{array}\right]$

and the vector ${ }^{t+\Delta t} \mathbf{F}_{c}$ is obtained after assembling ${ }^{t+\Delta t} \mathbf{F}_{c}^{k}$ for all Gauss points.

\subsection{Constraint functions}

As mentioned earlier, we use regularized constraint functions that approximately satisfy conditions (6) and (12)-(14).

Let $\epsilon_{n}$ and $\epsilon_{\tau}$ be given real positive numbers, and

$\lambda_{\epsilon}(g)=\frac{\epsilon_{n}}{g}$

$\tau_{\epsilon}(v)=\frac{2}{\pi} \arctan \left(\frac{v}{\epsilon_{\tau}}\right)$

Table 1

Contact geometry definition

For each contactor Gauss point $k$ : Determine $m^{t}$

Determine $\mathbf{U}^{m_{k}}, \boldsymbol{\Theta}^{m_{k}}$ and $\boldsymbol{\Sigma}^{k}$

Calculate $r_{*}$

Calculate $\mathbf{n}_{k}, \mathbf{s}_{k}$ and $g$

Calculate $\partial_{\mathbf{U}} \mathbf{n}_{k}, \partial_{\mathbf{U}} \mathbf{s}_{k}$ and $\partial_{\mathbf{U}} g$ 
which define curves in the plane $(g, \lambda)$ and $(v, \tau)$, respectively. The function $w_{n_{c}}(g, \lambda)$ is given by the surface generated by translating the straight line with direction $(1,1,1)$ along the curve defined by $\lambda_{\epsilon}(g)$. Similarly, $w_{s_{\epsilon}}(v, \tau)$ is obtained by translating the straight line with direction $(1,-1,1)$ along the curve $\tau_{\epsilon}(v)$.

According to these definitions, $w_{n_{\epsilon}}$ is given by

$w_{n_{\epsilon}}=\frac{\lambda+g}{2}-\sqrt{\left(\frac{g-\lambda}{2}\right)^{2}+\epsilon_{n}}$

and $w_{s_{\epsilon}}$ is defined implicitly by

$\tau+w_{s_{\epsilon}}-\frac{2}{\pi} \arctan \left(\frac{v-w_{s_{\epsilon}}}{\epsilon_{\tau}}\right)=0$

Note that the functions defined by Eqs. (139) and (140) are $C^{\infty}$ for any $\epsilon_{n}, \epsilon_{\tau}>0$. Furthermore, in the limit of $\epsilon_{n}, \epsilon_{\tau} \rightarrow 0$ they satisfy exactly conditions (6) and (12)-(14). The particular functions presented here are not the only possible options but are chosen based on considerations of numerical efficiency.

\subsection{Consistent tangent matrix}

In order to have good convergence properties in the Newton-Raphson scheme used to solve the resulting non-linear Eqs. (80)-(83), it is important to use the consistent tangent matrix or a close approximation thereof. Concentrating only on the stiffness matrix terms we have for a typical Newton-Raphson iteration

$$
\begin{aligned}
& +\left[\begin{array}{llll}
\partial_{\mathbf{U}} \mathbf{F}_{u}+\partial_{\mathbf{U}} \mathbf{R}_{c} & \partial_{\boldsymbol{\Theta}} \mathbf{F}_{u}+\partial_{\boldsymbol{\Theta}} \mathbf{R}_{c} & \partial_{\mathbf{P}} \mathbf{F}_{u} & \partial_{\boldsymbol{\Sigma}} \mathbf{R}_{c} \\
\partial_{\mathbf{U}} \mathbf{Q}_{\theta}+\partial_{\mathbf{U}} \mathbf{Q}_{c} & \partial_{\boldsymbol{\Theta}} \mathbf{Q}_{\theta}+\partial_{\boldsymbol{\Theta}} \mathbf{Q}_{c} & 0 & \partial_{\boldsymbol{\Sigma}} \mathbf{Q}_{c} \\
\partial_{\mathbf{U}} \mathbf{F}_{p} & \partial_{\boldsymbol{\Theta}} \mathbf{F}_{p} & \partial_{\mathbf{P}} \mathbf{F}_{p} & 0 \\
\partial_{\mathbf{U}} \mathbf{F}_{c} & \partial_{\boldsymbol{\Theta}} \mathbf{F}_{\mathbf{c}} & 0 & \partial_{\boldsymbol{\Sigma}} \mathbf{F}_{c}
\end{array}\right] \\
& \times\left\{\begin{array}{c}
\Delta \mathbf{U} \\
\Delta \boldsymbol{\Theta} \\
\Delta \mathbf{P} \\
\Delta \boldsymbol{\Sigma}
\end{array}\right\}=\cdots \\
&
\end{aligned}
$$

where we do not show the usual terms [1], and we have dropped the superscripts for ease of notation. We discuss in this section the derivatives of the terms arising due to contact, namely, $\mathbf{R}_{c}, \mathbf{Q}_{c}$ and $\mathbf{F}_{c}$. Differentiating $\mathbf{R}_{c}^{k}$ given in Eq. (134) we have,

$$
\partial_{\mathbf{U}} \mathbf{R}_{c}^{k}=\left[\begin{array}{c}
-\left\{\left(\lambda \partial_{\mathbf{U}} \mathbf{n}+\partial_{\mathbf{U}} t_{s} \mathbf{s}+t_{s} \partial_{\mathbf{U}} \mathbf{s}\right) \operatorname{det}\right. \\
\left.+\left(\lambda \mathbf{n}+t_{s} \mathbf{s}\right) \partial_{\mathbf{U}} \operatorname{det}\right\} h_{i} w \\
\left\{\left(\lambda \partial_{\mathbf{U}} \mathbf{n}+\partial_{\mathbf{U}} t_{s} \mathbf{s}+t_{s} \partial \mathbf{U} \mathbf{s}\right) h_{j}^{*} \operatorname{det}\right. \\
\left.\quad+\left(\lambda \mathbf{n}+t_{s} \mathbf{s}\right)\left(\partial_{\mathbf{U}} h_{j}^{*} \operatorname{det}+h_{j}^{*} \partial_{\mathbf{U}} \operatorname{det}\right)\right\} w
\end{array}\right]
$$

$\partial_{\boldsymbol{\Theta}} \mathbf{R}_{\mathrm{c}}^{k}=\left[\begin{array}{l}-\partial_{\boldsymbol{\Theta}} t_{s} \mathbf{s} h_{i} \operatorname{det} w \\ \partial_{\boldsymbol{\Theta}} t_{s} \mathbf{s} h_{j}^{*} \operatorname{det} w\end{array}\right]$

$\partial_{\lambda} \mathbf{R}_{c}^{k}=\left[\begin{array}{l}-\left(\mathbf{n}+\partial_{\lambda} t_{s} \mathbf{s}\right) h_{i} \operatorname{det} w \\ \left(\mathbf{n}+\partial_{\lambda} t_{s} \mathbf{s}\right) h_{j}^{*} \operatorname{det} w\end{array}\right]$

$\partial_{\tau} \mathbf{R}_{c}^{k}=\left[\begin{array}{l}-\partial_{\tau} t_{s} \mathbf{s} h_{i} \operatorname{det} w \\ \partial_{\tau} t_{s} \mathbf{s} h_{j}^{*} \operatorname{det} w\end{array}\right]$

In the same way we have for $\mathbf{Q}_{c}^{k}$

$\partial_{\mathbf{U}} \mathbf{Q}_{c}^{k}=\left[\begin{array}{c}-\left\{\left(\hat{h} \partial_{\mathbf{U}} \Delta \theta^{I J}+\partial_{\mathbf{U}} q_{G}^{I J} \frac{k_{I}}{\xi_{I}}\right) \operatorname{det}\right. \\ \left.+\left(\hat{h} \Delta \theta^{I J}+q_{G}^{I J} \frac{k_{I}}{\xi_{I}}\right) \partial_{\mathbf{U}} \operatorname{det}\right\} h_{i} w \\ \left\{\left(\hat{h} \partial_{\mathbf{U}} \Delta \theta^{I J}-\partial_{\mathbf{U}} q_{G}^{I J} \frac{k_{J}}{\xi_{J}}\right) h_{j}^{*} \operatorname{det}\right. \\ \left.+\left(\hat{h} \Delta \theta^{I J}-q_{G}^{I J} \frac{k_{J}}{\xi_{J}}\right)\left(\partial_{\mathbf{U}} h_{j}^{*} \operatorname{det}+h_{j}^{*} \partial_{\mathbf{U}} \operatorname{det}\right)\right\} w\end{array}\right]$

$\partial_{\Theta} \mathbf{Q}_{c}^{k}=\left[\begin{array}{c}-\left(\hat{h} \partial_{\Theta} \Delta \theta^{I J}+\partial_{\Theta} q_{G}^{I J} \frac{k_{I}}{\xi_{I}}\right) h_{i} \operatorname{det} w \\ \left(\hat{h} \partial_{\Theta} \Delta \theta^{I J}-\partial_{\Theta} q_{G}^{I J} \frac{k_{J}}{\xi_{J}}\right) h_{j}^{*} \operatorname{det} w\end{array}\right]$

$\partial_{\lambda} \mathbf{Q}_{c}^{k}=\left[\begin{array}{l}-\left(\partial_{\lambda} \hat{h} \Delta \theta^{I J}+\partial_{\lambda} q_{G}^{I J} \frac{k_{I}}{\xi_{I}}\right) h_{i} \operatorname{det} w \\ \left(\partial_{\lambda} \hat{h} \Delta \theta^{I J}-\partial_{\lambda} q_{G}^{I J} \frac{k_{J}}{\xi_{J}}\right) h_{j}^{*} \operatorname{det} w\end{array}\right]$

$\partial_{\tau} \mathbf{Q}_{c}^{k}=\left[\begin{array}{c}\partial_{\tau}\left(-q_{G}^{I J} \frac{k_{I}}{\xi_{I}} h_{i} \operatorname{det} w\right) \\ \partial_{\tau}\left(-q_{G}^{I J} \frac{k_{J}}{\xi_{J}} h_{j}^{*} \operatorname{det} w\right)\end{array}\right]$

and for the vector $\mathbf{F}_{c}^{k}$,

$\partial_{\mathbf{U}} \mathbf{F}_{c}^{k}=\left[\begin{array}{l}\partial_{g} w_{n_{\epsilon}} \partial_{\mathbf{U}} g \\ \partial_{v} w_{s_{\epsilon}} \partial_{\mathbf{U}} v\end{array}\right]$ 
Table 2

Coupled thermo-mechanical contact

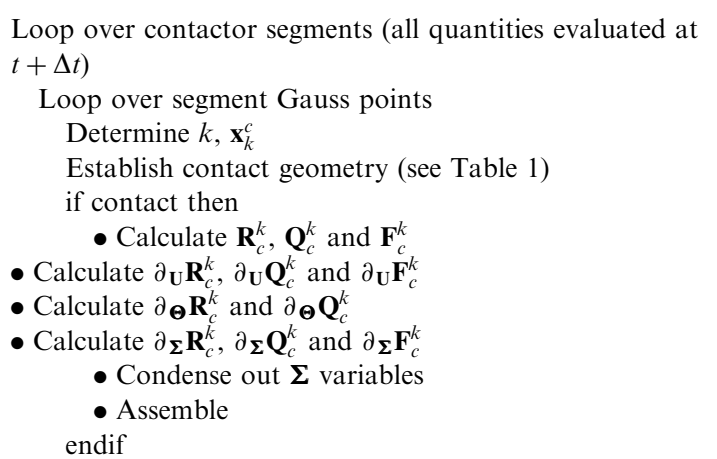

End loop over segment Gauss points

End loop over contactor segments

Solve linear system for iteration $i$

(denoting the forcing vectors by $\mathbf{F}$ and $\mathbf{Q}$ and assuming $u / p$

elements are used [1])

$\left[\begin{array}{cc}\partial_{\mathbf{U}} \mathbf{F}^{(i-1)} & \partial_{\mathbf{\Theta}} \mathbf{F}^{(i-1)} \\ \partial_{\mathbf{U}} \mathbf{Q}^{(i-1)} & \partial_{\mathbf{\Theta}} \mathbf{Q}^{(i-1)}\end{array}\right]\left\{\begin{array}{c}\Delta \mathbf{U}^{(i)} \\ \Delta \boldsymbol{\Theta}^{(i)}\end{array}\right\}=\left\{\begin{array}{l}\mathbf{F}^{(i-1)} \\ \mathbf{Q}^{(i-1)}\end{array}\right\}$

Update displacements: ${ }^{t+\Delta t} \mathbf{U}^{(i)}={ }^{t+\Delta t} \mathbf{U}^{(i-1)}+\Delta \mathbf{U}^{(i)}$

Update temperatures: ${ }^{t+\Delta t} \boldsymbol{\Theta}^{(i)}={ }^{t+\Delta t} \boldsymbol{\Theta}^{(i-1)}+\Delta \boldsymbol{\Theta}^{(i)}$

$\partial_{\boldsymbol{\Theta}} \mathbf{F}_{c}^{k}=\left[\begin{array}{l}0 \\ 0\end{array}\right]$

$\partial_{\lambda} \mathbf{F}_{c}^{k}=\left[\begin{array}{l}\partial_{\lambda} w_{n_{\epsilon}} \\ 0\end{array}\right]$

$\partial_{\tau} \mathbf{F}_{c}^{k}=\left[\begin{array}{l}0 \\ \partial_{\tau} w_{s_{\epsilon}}\end{array}\right]$

In the above, we have

$$
\begin{aligned}
& \partial_{\mathbf{U}} t_{s}=\tau \partial_{\mathbf{U}} \zeta \\
& \partial_{\boldsymbol{\Theta}} t_{s}=\tau \partial_{\boldsymbol{\Theta}} \zeta \\
& \partial_{\lambda} t_{s}=\tau \partial_{\lambda} \zeta \\
& \partial_{\tau} t_{s}=\zeta \\
& \partial_{\mathbf{U}} q_{G}^{I J}=\gamma\left(\partial_{\mathbf{U}} t_{s} v+t_{s} \partial_{\mathbf{U}} v\right) \\
& \partial_{\boldsymbol{\Theta}} q_{G}^{I J}=\gamma \partial_{\mathbf{\Theta}} t_{s} v \\
& \partial_{\lambda} q_{G}^{I J}=\gamma \partial_{\lambda} t_{s} v
\end{aligned}
$$

$\partial_{\tau} q_{G}^{I J}=\gamma \partial_{\tau} t_{s} v$

$\partial_{\lambda} \hat{h}=1.45 \frac{\bar{k}}{H} \frac{|\tan \delta|}{\sigma}$

and

$\partial_{\mathbf{U}} h_{j}^{*}=\left.\partial_{r} h_{j}^{*}\right|_{r=r^{*}} \partial_{\mathbf{U}} r^{*}$

The derivatives of $v$ with respect to $\mathbf{U}$ differ depending on whether we consider the contactor or the target body; hence, we separate $\partial_{\mathbf{U}} v$ into

$\partial_{\mathbf{u}_{p}^{c} v}=-\frac{\left.h_{p}\right|_{r=r_{k}}}{\Delta t} \cdot \mathbf{s}^{J}+\left(\mathbf{v}^{J}-\mathbf{v}^{I}\right) \cdot \partial_{\mathbf{u}_{p}^{c}} \mathbf{s}^{J}$

$\partial_{\mathbf{u}_{q}^{t}} v=\frac{\left.h_{q}\right|_{r=r^{*}}}{\Delta t} \cdot \mathbf{s}^{J}+\left(\mathbf{v}^{J}-\mathbf{v}^{I}\right) \cdot \partial_{\mathbf{u}_{q}^{t}} \mathbf{S}^{J}$

Similarly, $\partial_{\boldsymbol{\Theta}}\left(\Delta \theta^{I J}\right)$ is given by

$\partial_{\theta_{p}^{c}}\left(\Delta \theta^{I J}\right)=-\left.h_{p}\right|_{r=r_{k}}$

$\partial_{\theta_{q}^{t}}\left(\Delta \theta^{I J}\right)=\left.h_{q}^{*}\right|_{r=r^{*}}$

and

$\partial_{\mathbf{U}}\left(\Delta \theta^{I J}\right)=\partial_{\mathbf{U}} h_{q}^{*}$

The Jacobian determinant only depends on the contactor element nodal displacements

$\partial_{\mathbf{u}_{p}^{c}} \operatorname{det}=\frac{\partial_{r} h_{p} \mathbf{x}_{k}^{c}}{\operatorname{det}}$

Note that in the actual implementation, it is not necessary to assemble either the vector ${ }^{t+\Delta t} \mathbf{F}_{c}$ or the stiffness submatrices $\partial_{\mathbf{U}}{ }^{t+\Delta t} \mathbf{F}_{c}, \partial_{\boldsymbol{\Theta}^{t+\Delta t}} \mathbf{F}_{c}$ and $\partial_{\boldsymbol{\Sigma}}{ }^{t+\Delta t} \mathbf{F}_{c}$. Since the contact variables are calculated at the Gauss points of the contactor elements, they do not couple between elements and therefore, in principle, can be condensed out at the contact element level during the assembling procedure (where to avoid ill-conditioning a proper scale on $\lambda$ should be used). Note also that $\partial_{\boldsymbol{\Sigma}}{ }^{t+\Delta t} \mathbf{F}_{c}$ results in a diagonal matrix and the inversion is trivial. Therefore, the number of algebraic equations to solve is the same as for the penalty method.

Table 2 presents a complete algorithm for the coupled thermo-mechanical contact problem.

In our earlier work we imposed the contact constraints at the nodal points [1,33]. Using the Gauss point positions raises questions as to whether imposing the constraints will always result into stability of the solution and in optimal accuracy. To address these questions a mathematical analysis is necessary, but we may note that in our numerical solutions, presented 
next, we did not observe any solution abnormalities or special difficulties.

\section{Numerical examples}

We present in this section some numerical results to illustrate the procedures described in this paper.

\subsection{Block heating due to frictional contact}

An elastic body in plane strain conditions slides over a rigid block which is considered to be fixed. The system is depicted in Fig. 6. Initially, the temperature of both bodies is the ambient temperature. All the surfaces are considered to be adiabatic. The body moves from left to right at a prescribed velocity $v=1000$ $\mathrm{mm} / \mathrm{s}$. A pressure $p$ is applied at the top of the elastic body. The material properties are given in Table 3 . Inertia effects are neglected.

The body is moved up to its final position within a time $t=0.00375 \mathrm{~s}$ and then is left to rest until the tem-

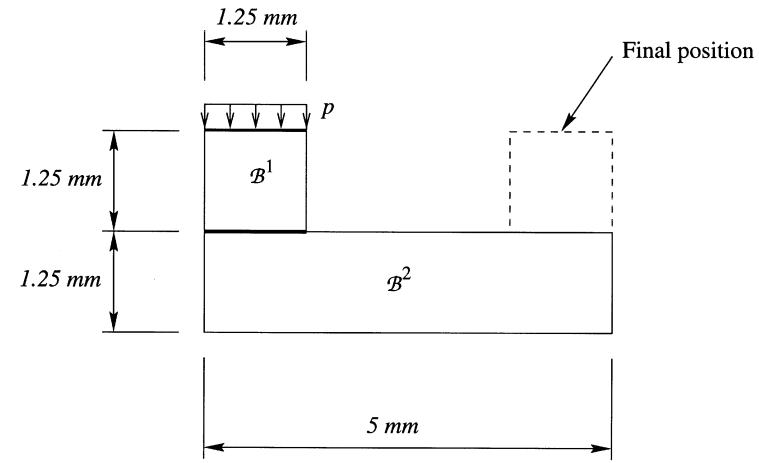

Fig. 6. Block heating due to frictional contact. Model problem.

perature becomes uniform in the whole system, i.e., a steady state is reached. Fig. 7 shows temperature band plots at different positions of the body during its motion.

Since all surfaces are insulated, in our mathematical model all energy generated at the interface due to fric-
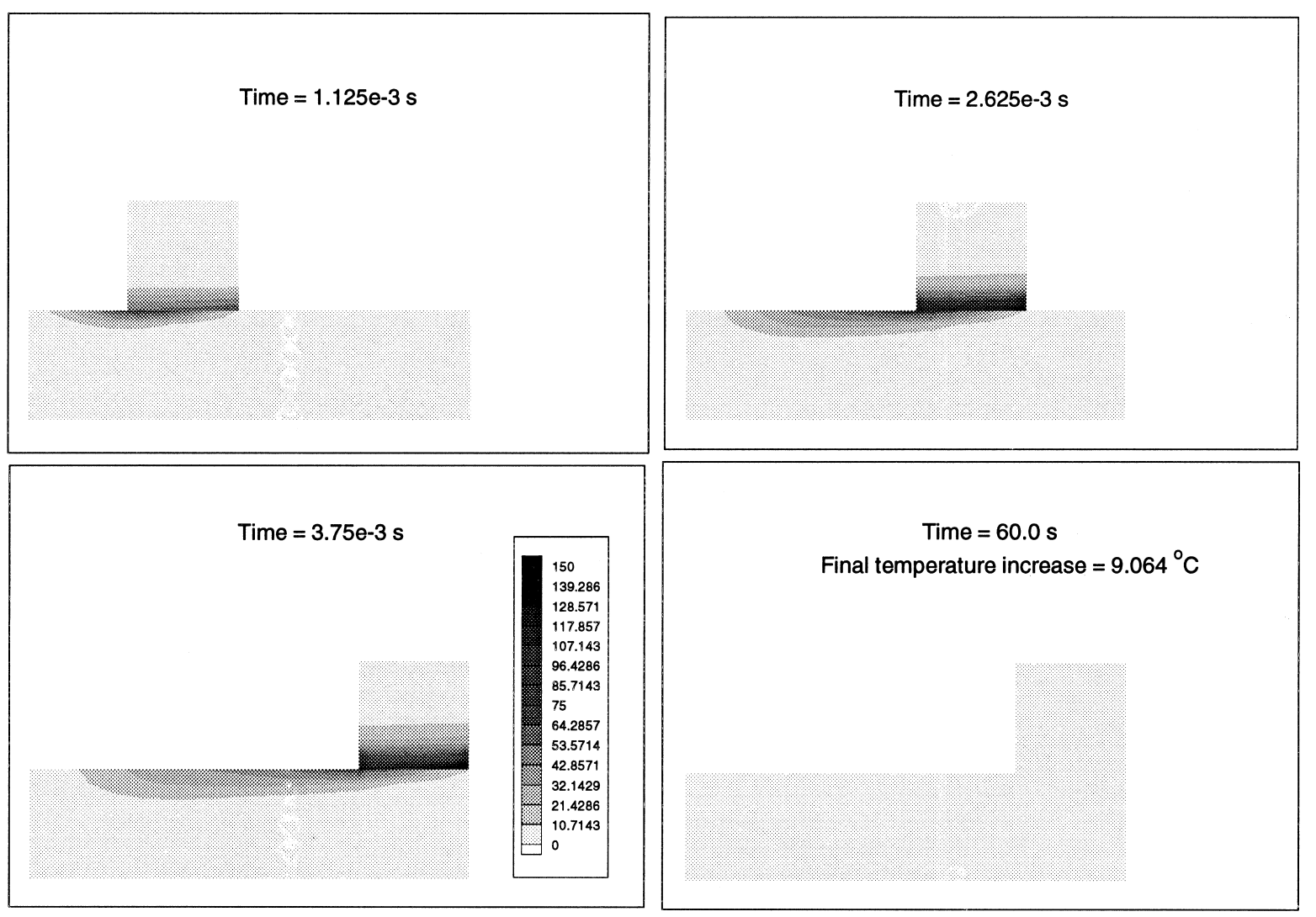

Fig. 7. Block heating due to frictional contact at $p=0.3 \mathrm{kN} / \mathrm{mm}^{2}$ using the friction law given in Eq. (10). Contours of temperature increase. 
Table 3

Block heating due to frictional contact. Material properties

\begin{tabular}{lll}
\hline Bulk modulus & $\kappa$ & $166667 \mathrm{~N} / \mathrm{mm}^{2}$ \\
Shear modulus & $G$ & $76920 \mathrm{~N} / \mathrm{mm}^{2}$ \\
Density & $\rho$ & $7.8 \mathrm{E}-9 \mathrm{Ns}^{2} / \mathrm{mm}^{4}$ \\
Expansion coefficient & $\alpha$ & $1.0 \mathrm{E}-6 \mathrm{~K}^{-1}$ \\
Conductivity & $k$ & $45 \mathrm{~N} / \mathrm{sK}$ \\
Heat capacity & $c$ & $4.6 \mathrm{E}+8 \mathrm{~mm}^{2} / \mathrm{s}^{2} \mathrm{~K}$ \\
Heat loss coefficient & $\gamma$ & 1.0 \\
Surface characteristics & & \\
& $\delta$ & 0.160 \\
& $\sigma$ & 0.032 \\
& $\zeta^{*}$ & $0.105 \mathrm{~N} / \mathrm{mm}^{2}$ \\
& $\mu$ & 0.2
\end{tabular}

tion is transformed into heat in the bodies. The corresponding steady state increase in temperature can be easily calculated

$\theta_{\infty}=\frac{W_{\text {gen }}}{\rho c V}$

where $V$ is the total volume of the system and $W_{\text {gen }}$ is the energy generated by friction,

$W_{\text {gen }}=\iint v t_{s} \mathrm{~d} t \mathrm{~d} x=u t_{s} A$
Table 4

Block in a converging channel. Material properties

Elasto-plastic material

Bulk modulus

Shear modulus

Flow stress

Hardening modulus

Density

Expansion coefficient

Conductivity

Heat capacity

Flow stress softening

Heat loss coefficient

Surface characteristics

Contact parameters

$\begin{array}{ll}\kappa & 166667 \mathrm{~N} / \mathrm{mm}^{2} \\ G & 76920 \mathrm{~N} / \mathrm{mm}^{2} \\ \sigma_{y 0} & 284.4 \mathrm{~N} / \mathrm{mm}^{2} \\ h & 270 \mathrm{~N} / \mathrm{mm}^{2} \\ \rho & 7.8 \mathrm{E}-9 \mathrm{Ns}^{2} / \mathrm{mm}^{4} \\ \alpha & 12.0 \mathrm{E}-6 \mathrm{~K}^{-1} \\ k & 45.0 \mathrm{~N} / \mathrm{sK}^{2} \mathrm{~m}^{2} \mathrm{~K} \\ c & 4.6 \mathrm{E}+8 \mathrm{~mm}^{-1} \\ w_{0} & 3.0 \mathrm{E}-3 \mathrm{~K}^{-1} \\ \gamma & 1.0 \\ \delta & \\ \sigma & 0.160 \\ \mu & 0.0302 \\ & 0.15\end{array}$

$\begin{array}{ccc}\epsilon_{n} & 1.0 \mathrm{E}-7 \\ c_{t} & 1.0 \mathrm{E}-6\end{array}$

$1.0 \mathrm{E}-6$

where $x$ is a coordinate measuring the distance travelled. Fig. 8 shows the temperature increase for the applied pressure. A comparison of the results obtained when using Coulomb's law and the law in Eq. (10) shows that both models predict almost the same results for low pressures but at large pressure values, the use of Coulomb's law results into higher temperatures.

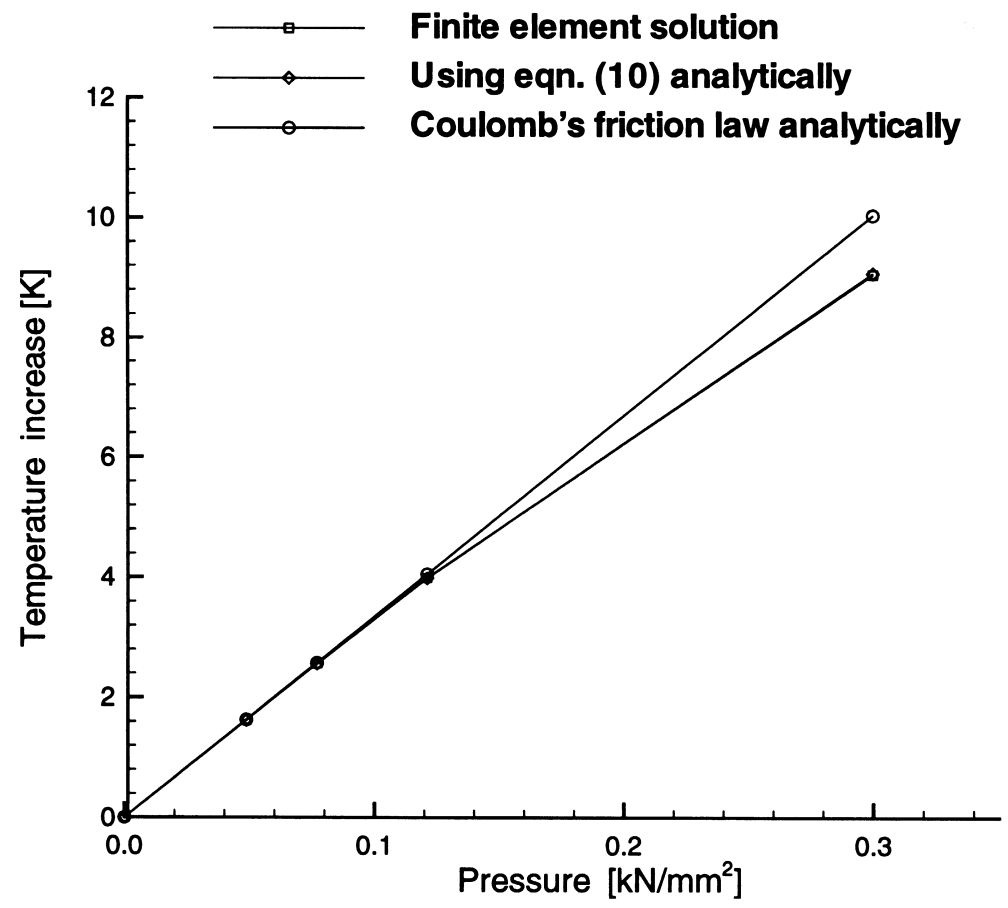

Fig. 8. Block heating due to frictional contact. Temperature increase vs. pressure at final time. 


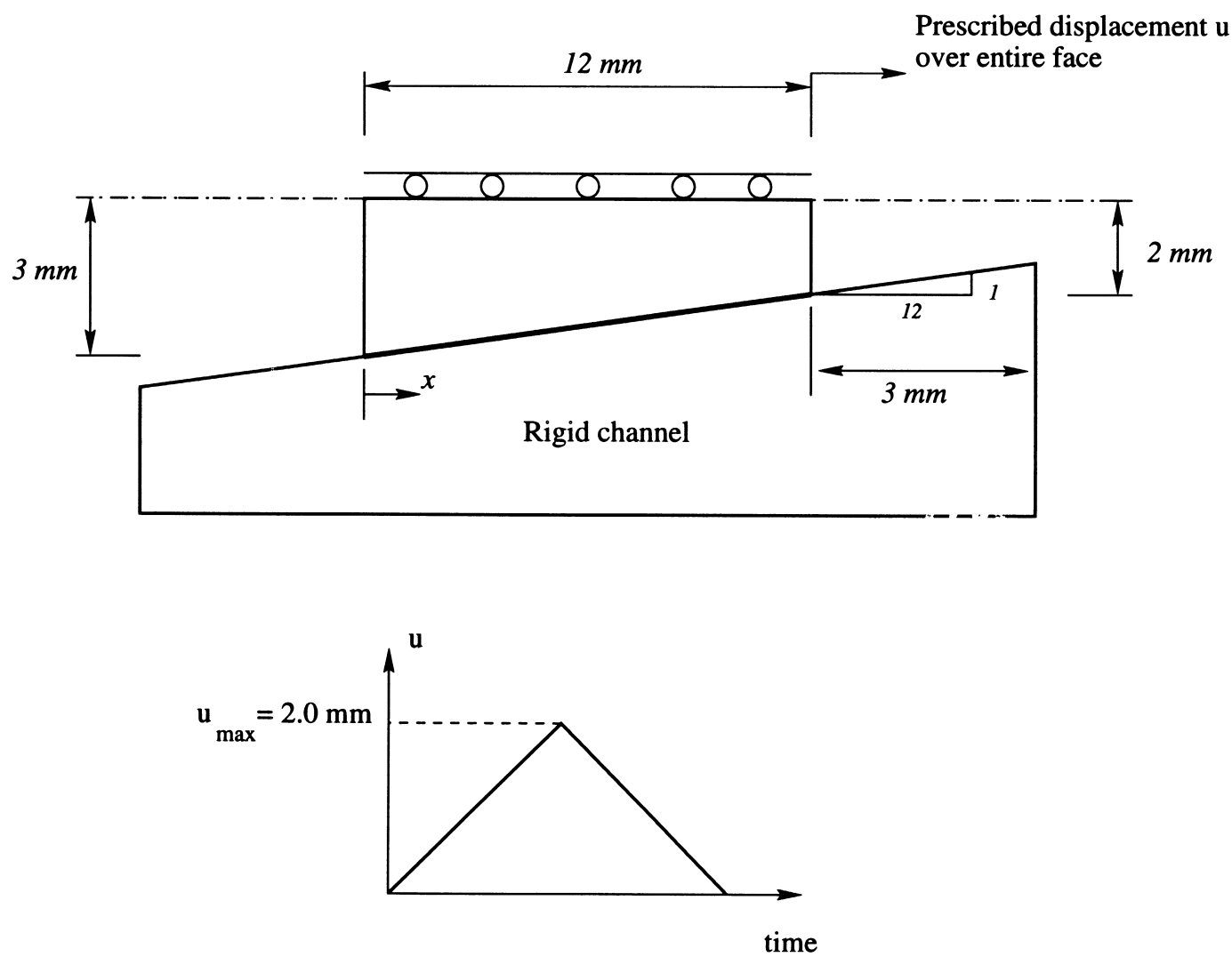

Fig. 9. Block in a converging channel. Plane strain model problem.

\subsection{Block in a converging channel}

A block of rubber material is confined to move in a converging rigid channel. We assume plane strain conditions. A similar problem under plane stress conditions was analyzed by Bathe and Chaudhary [14], and Bathe and Bouzinov [33]. This problem represents a valuable test for a contact algorithm since the essential features and solution difficulties of this problem are frequently encountered in actual practical applications. The material parameters used are $C_{1}=25.0$ $\mathrm{N} / \mathrm{mm}^{2}, C_{2}=7.0 \mathrm{~N} / \mathrm{mm}^{2}$ and the bulk modulus $\kappa=$ $1000.0 \mathrm{~N} / \mathrm{mm}^{2}$. We use 9-node elements to analyze this problem. The model and mesh considered are shown in Figs. 9 and 10, respectively. The right face of the block is subjected to a prescribed displacement $\Delta$. Inertia effects are neglected.

Fig. 11 shows the distribution of the normal contact tractions at two different displacements for the frictionless case using the Mooney-Rivlin material model. We also present a comparison against a standard approach. Note that, small oscillations are present using the standard approach whereas no oscillations appear when using the algorithm based on Gauss point quantities. With friction present, $\mu=0.15$, we move the block to the right and then back to the original position. Figs. 12 and 13 give the distributions of the normal and tangential contact tractions at four levels of displacement application. The results are plotted with respect to the original coordinates.

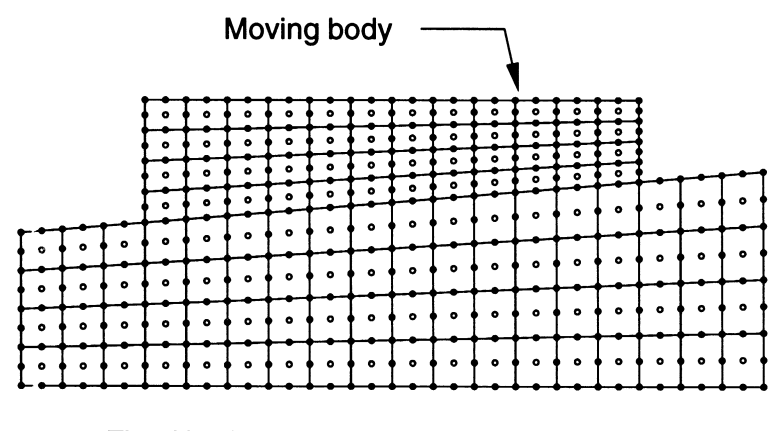

Fixed body

Fig. 10. Block in a converging channel. Finite element model considerd using $9 / 3$ element (with 9 nodes for the displacement interpolation and 3 pressure degrees of freedom [1]). 


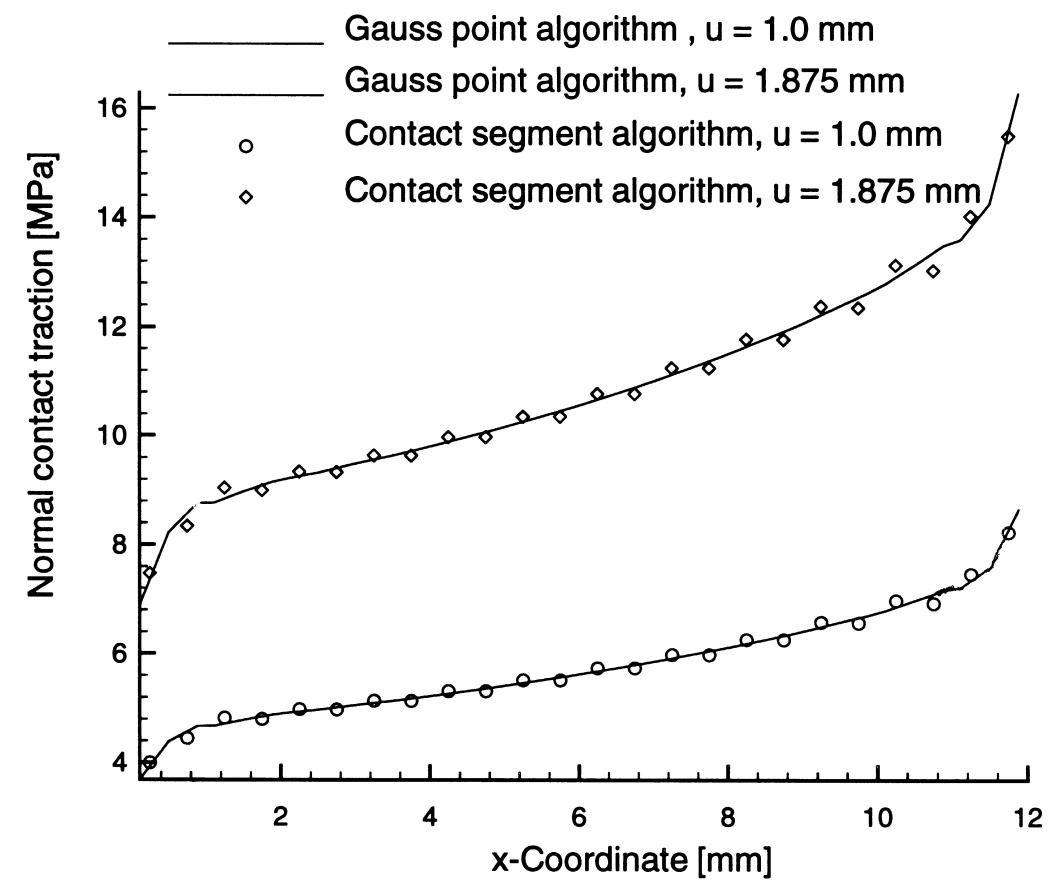

Fig. 11. Contact tractions for the frictionless case. Mooney-Riviln material.

\subsection{Extrusion of a steel block}

We analyze in this example the forming of a steel block. In this solution we seek the response of the steel block when the block is thermo-mechanically fully coupled to the channel. The same finite element discretization as in the previous example is considered here (see Figs. 9 and 10). The thermo-mechanical material

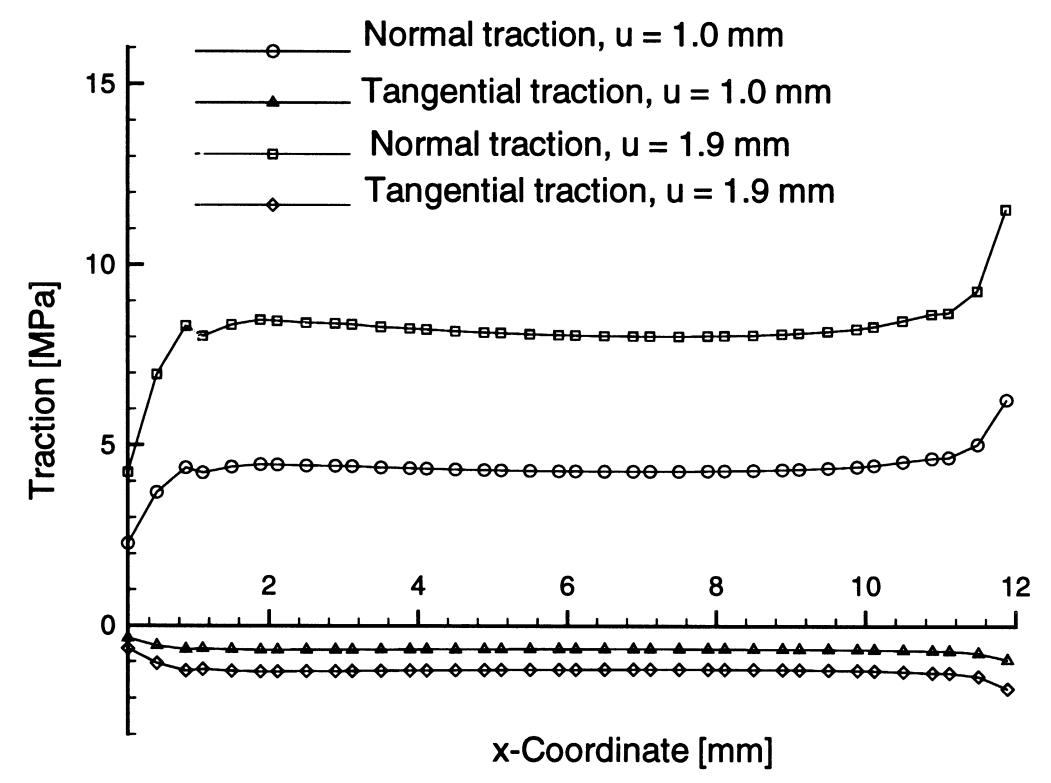

Fig. 12. Contact traction when $\mu=0.15$. Block is moving to the right. Mooney-Rivlin material. 


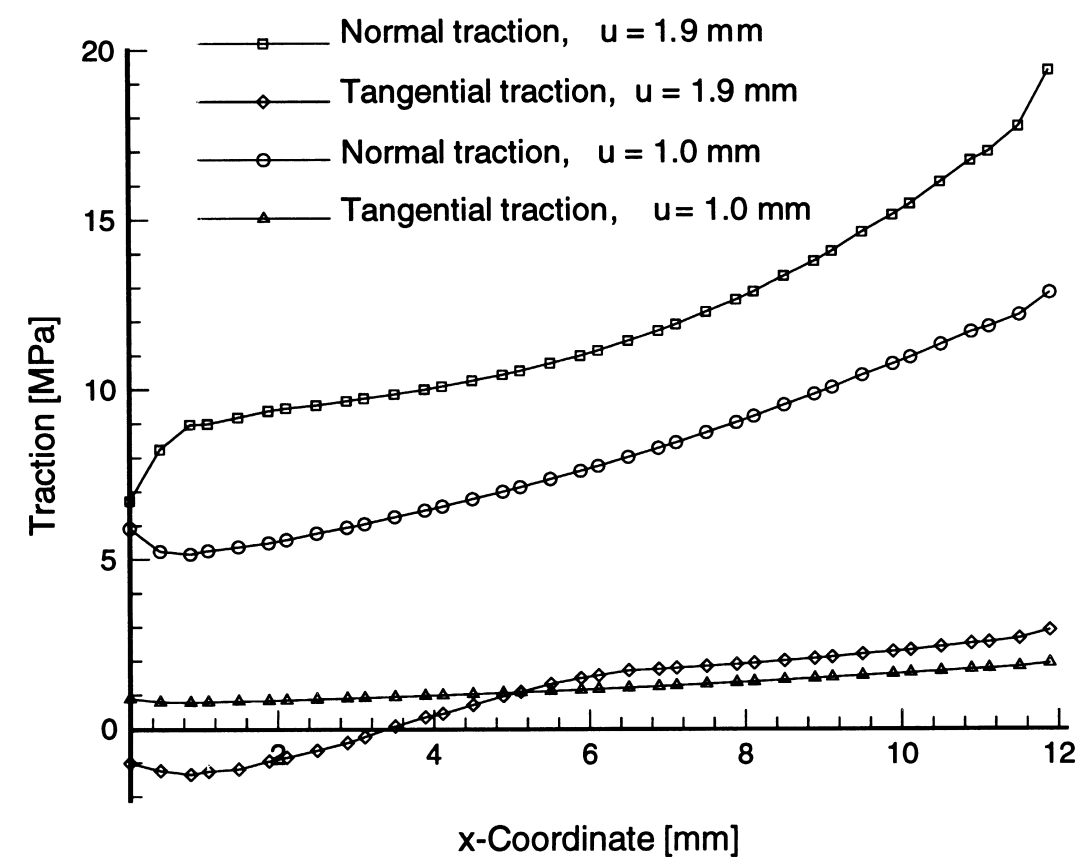

Fig. 13. Contact traction when $\mu=0.15$. Block is moving to the left. Mooney-Rivlin material.

properties are given in Table 4. Adiabatic boundary conditions were considered all around the complete model (including the steel block and die).

The block is now moved at $v=100 \mathrm{~m} / \mathrm{s}$ until a total prescribed displacement $u=16 \mathrm{~mm}$ is reached. Inertia effects are neglected.

Figs. 14 and 15 show the final configuration of the body and a contour temperature plot after the total final displacement is reached. All the temperature rise is due to plastic and frictional heat generation. The large temperature rise at the contact interface shows the importance of the amount of heat generated by friction.
The applied force as a function of the applied displacement is given in Fig. 16. A comparison between a purely mechanical and a thermo-mechanical simulation is also given. First, the load increases as the displacements are applied. Then, as the body is moved further and part of it loses contact with the fixed body, the load decreases. In the thermo-mechanical case, due to the softening effect of the temperature, the load is decreasing faster than in the purely mechanical analysis. A difference of about $30 \%$ is obtained in the force magnitude between the mechanical and thermo-mechanical analyses after the displacement $u$ reaches its maximum.

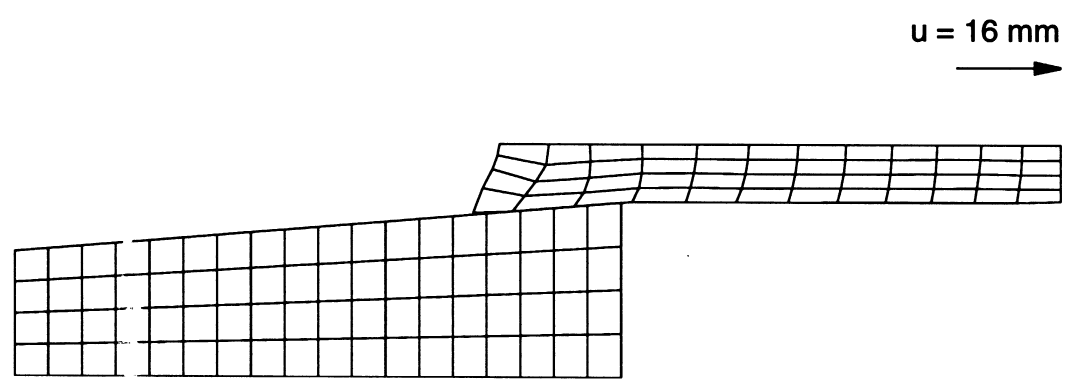

Fig. 14. Final configuration when $u=16 \mathrm{~mm}$. 

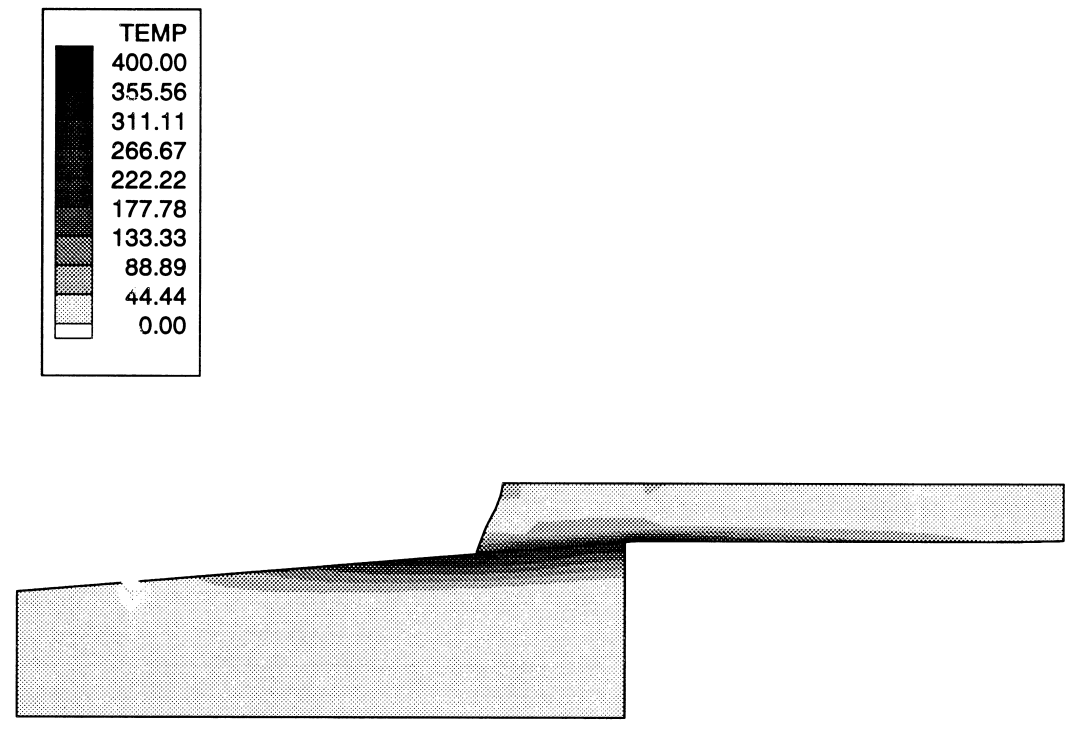

Fig. 15. Band plot of temperature rise at $u=16 \mathrm{~mm}$.

\section{Conclusions}

In this paper we presented a solution procedure for the analysis of thermo-mechanical conditions of solids in contact. The thermal and stress conditions are fully coupled in the analysis. The advances given in the paper primarily relate to the modeling and solution of the contact conditions. A quite general contact constitutive relation is employed and incorporated into the constraint function algorithm. The solution procedure presented here is promising, but clearly further studies regarding the accuracy of the mathematical modeling and solution are still needed.

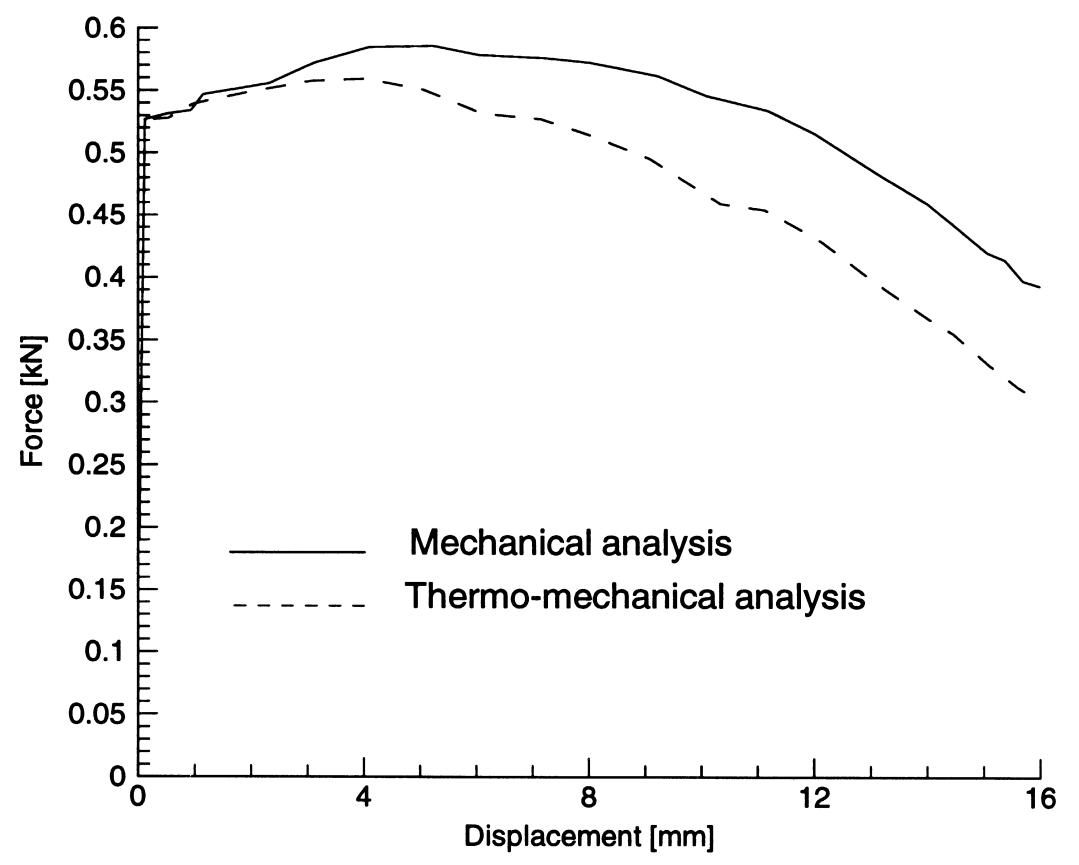

Fig. 16. Force vs displacement curves. 


\section{Acknowledgements}

We are grateful for the support provided by the Rocca fellowship for D. Pantuso's studies at M.I.T.

\section{Appendix}

We give in this appendix a derivation of Eq. (56). According to Eq. (52), the rate of change of the contact traction is

$\dot{\mathbf{t}}=\mathbf{A} \overline{\mathbf{v}}-k_{T} \overline{\mathbf{v}}_{T}^{s}$

Let us consider that the bodies are slipping $(\chi=1)$. Hence, we have $f=0$ in Eq. (55) and

$\dot{f}\left(t_{s}, \zeta\right)=\dot{t}_{s}-\dot{\zeta}=0$

which implies

$\dot{t}_{s}=\dot{\zeta}=h_{N} \dot{\lambda}+h_{T} v_{T}^{s}$

The normal to the slip surface is given by

$\partial_{\mathbf{t}} f=\overline{\mathbf{s}}=\mathbf{s}-h_{N} \mathbf{n}$

Using Eqs. (A3) and (A4), we have that

$v^{s}=\frac{1}{h_{T}+k_{T}} \overline{\mathbf{s}} \cdot\{\mathbf{A v}\}$

where $v^{s}$ is the magnitude of the slipping part of the relative tangential velocity, namely $v^{s}=v-v^{a}$. Using Eqs. (54) and (A1) to (A5) we can write the rate of change of the contact traction as

$\dot{\mathbf{t}}=\mathbf{A} \overline{\mathbf{v}}-\frac{k_{T}}{k_{T}+h_{T}}\left[\left(\mathbf{s}-h_{N} \mathbf{n}\right) \cdot \mathbf{A} \overline{\mathbf{v}}\right] \mathbf{s}$

Replacing A given by Eq. (53) in the second term of Eq. (A6) and after some algebraic manipulations, we obtain Eq. (56).

\section{References}

[1] Bathe KJ. Finite element procedures. Englewood Cliffs, New Jersey: Prentice Hall, 1996.

[2] Rabinowicz E. Friction and wear of materials. New York: Wiley, 1965.

[3] Suh NP. Tribophysics. Englewood Cliffs: Prentice Hall, 1986.

[4] Oden JT, Martins JAC. Models for computational methods for dynamic friction phenomena. Computer Methods in Applied Mechanics and Engineering 1985;52:527-634.

[5] Pantuso D, Bathe KJ. Finite element analysis of thermoelasto-plastic solids in contact. In: Owen DRJ, Onate E, editors. Computational plasticity, CIMNE, 1997, p. 72 87.

[6] Eterovic AL, Bathe KJ. On large strain elasto-plastic analysis with frictional contact conditions. In: Proceedings of the Conference on Numerical Methods in Applied Science and Industry. Politecnico di Torino, 1990. p. 379-463.

[7] Eterovic AL, Bathe KJ. On the treatment of inequality constraints arising from contact conditions in finite element analysis. Computers and Structures 1991;40:203-9.

[8] Anand L. A constitutive model for interface friction. Computational Mechanics 1993;12:123-97.

[9] Anand L, Tong W. A constitutive model for friction in forming. Annals of the CIRP 1993;42(1):361-6.

[10] Wriggers P, Miehe C. Contact constraints within coupled thermomechanical analysis - a finite element model. Computer Methods in Applied Mechanics and Engineering 1994;113:301-19.

[11] Miles MP, Fourment L, Chenot JL. Finite element calculation of thermal coupling between workpiece and tools in forging. Engineering Computations 1995;12:687-705.

[12] Zavarise G, Wriggers P, Schrefler BA. On augmented Lagrangian algorithms for thermomechanical contact problems with friction. International Journal for Numerical Methods in Engineering 1995;38:2929-49.

[13] Bathe KJ, Chaudhary AB. On finite element analysis of large deformation frictional contact problems. In: Proceedings of the 7th Invitational Symposium on the Unification of Finite Elements, Finite Differences and Calculus of Variations. 1984. p. 165-89.

[14] Bathe KJ, Chaudhary AB. A solution method for planar and axisymmetric contact problems. International Journal for Numerical Methods in Engineering 1985;21:65-88.

[15] Oden JT. Exterior penalty methods for contact problems in elasticity. In: Wunderlich W, Stein E, Bathe KJ, editors. Nonlinear finite element analysis in structural mechanics. New York: Springer-Verlag, 1980.

[16] Kikuchi N. A smoothing technique for reduced integration penalty methods in contact problems. International Journal for Numerical Methods in Engineering 1982;18:343-50.

[17] Simo JC, Wriggers P, Taylor RL. A perturbed Lagrangian formulation for the finite element solution of contact problems. Computer Methods in Applied Mechanics and Engineering 1985;50:163-80.

[18] Simo JC, Laursen TA. An augmented Lagrangian treatment of contact problems involving friction. Computers and Structures 1992;42:97-116.

[19] Wriggers P, Simo JC, Taylor RL. Penalty and augmented Lagrangian formulations for contact problems. In: Middleton J, Pande GN, editors. Proceedings of the International Conference on Numerical Methods in Engineering - Theory and Applications, NUMETA'85, 1985. p. $97-106$

[20] Lehmann Th. General frame for the definition of constitutive laws for large non-isothermic elastic-plastic and elastic-viscoplastic deformations. Lehmann Th, editor. The Constitutive Law in Thermoplasticity, CISM Courses and Lectures No 281, 1984. p. 379-463. 
[21] Anand L. Constitutive equations for hot-working of metals. International Journal of Plasticity 1985;1:213-31.

[22] Mikic BB. Thermal contact conductance: theoretical considerations. International Journal of Heat and Mass Transfer 1974;17:205-14.

[23] Truesdell C, Noll W. The nonlinear field theories of mechanics, 2nd ed. New York: Springer-Verlag, 1992.

[24] Bathe KJ, Zhang H, Ji S. Finite element analysis of fluid flows fully coupled with structural interactions. Computers and Structures 1999;72:1-16.

[25] Asaro RJ. Micromechanics of crystals and polycrystals. Adv. Applied Mechanics. 1983;23(1).

[26] Simo JC. On the computational significance of the intermediate configuration and hyperelastic stress relations in finite deformation elastoplasticity. Mech Mater 1985;4:439-51.

[27] Carslaw HS, Jaeger JC. Conduction of heat in solids. Oxford: Oxford University Press, 1947.

[28] Madhusudana CV, Fletcher LS. Contact heat transferthe last decade. AIAA Journal 1986;24:510-23.

[29] Cheng JH, Kikuchi N. An incremental constitutive relation of unilateral contact friction for large deformation analysis. Journal of Applied Mechanics 1985;52:639-48.

[30] Sussman T, Bathe KJ. A finite element formulation for nonlinear incompressible elastic and inelastic analysis. Computers and Structures 1987;26(1/2):357-409.

[31] Pantuso D, Bathe KJ. A four-node quadrilateral mixedinterpolated element for solids and fluids. Mathematical Models and Methods in Applied Sciences 1995;5(8):1113-28.

[32] Pantuso D, Bathe KJ. On the stability of mixed finite elements in large strain analysis of incompressible solids. Finite Elements in Analysis and Design 1997;28:83-104.

[33] Bathe KJ, Bouzinov PA. On the constraint function method for contact problems. Computers and Structures 1997;64(5/6):1069-85.

[34] Atluri SN. Alternate stress and conjugate strain measures, and mixed variational formulations involving rigid rotations, for computational analysis of finitely deformed solids, with application to plates and shells: Part I - theory. Computers and Structures 1984;18:93116.

[35] Eterovic AL, Bathe KJ. A hyperelastic-based large strain elasto-plastic constitutive formulation with combined isotropic-kinematic hardening using the logarithmic stress and strain measures. International Journal for Numerical Methods in Engineering 1990;30:1099-114. 